\title{
Supersymmetry Parameter Analysis: SPA Convention and Project
}

\begin{abstract}
J.A. Aguilar-Saavedra ${ }^{1}$, A. Ali ${ }^{2}$, B.C. Allanach ${ }^{3}$, R. Arnowitt ${ }^{4}$, H.A. Baer ${ }^{5}$, J.A. Bagger ${ }^{6}$ C. Balazs $^{7 a}$, V. Barger ${ }^{8}$, M. Barnett ${ }^{9}$, A. Bartl ${ }^{10}$, M. Battaglia ${ }^{9}$, P. Bechtle ${ }^{11}$, G. Bélanger ${ }^{12}$, A. Belyaev ${ }^{13}$, E.L. Berger ${ }^{7}$, G. Blair ${ }^{14}$, E. Boos ${ }^{15}$, M. Carena ${ }^{16}$, S.Y. Choi ${ }^{17}$, F. Deppisch ${ }^{2}$, A. De Roeck ${ }^{18}$, K. Desch ${ }^{19}$, M.A. Diaz ${ }^{20}$, A. Djouadi ${ }^{21}$, B. Dutta ${ }^{4}$, S. Dutta ${ }^{22,11}$, H. Eberl $^{23}$, J. Ellis ${ }^{18}$, J. Erler ${ }^{24 b}$, H. Fraas ${ }^{25}$, A. Freitas ${ }^{26}$, T. Fritzsche ${ }^{27}$, R.M. Godbole ${ }^{28}$, G.J. Gounaris ${ }^{29}$, J. Guasch ${ }^{30}$, J. Gunion ${ }^{31}$, N. Haba ${ }^{32}$, H.E. Haber ${ }^{33}$, K. Hagiwara ${ }^{34}$, L. $\operatorname{Han}^{35}$, T. Han ${ }^{8}$, H.-J. He ${ }^{36}$, S. Heinemeyer ${ }^{18}$, S. Hesselbach ${ }^{37}$, K. Hidaka ${ }^{38}$, I. Hinchliffe ${ }^{9}$, M. Hirsch ${ }^{39}$, K. Hohenwarter-Sodek ${ }^{10}$, W. Hollik ${ }^{27}$, W.S. Hou ${ }^{40}$, T. Hurth ${ }^{18,11 ~ c}$, I. Jack ${ }^{41}$, Y. Jiang ${ }^{35}$, D.R.T. Jones ${ }^{41}$, J. Kalinowski ${ }^{42 d}$, T. Kamon ${ }^{4}$, G. Kane ${ }^{43}$, S.K. Kang ${ }^{44}$, T. Kernreiter ${ }^{10}$, W. Kilian ${ }^{2}$, C.S. Kim ${ }^{45}$, S.F. King ${ }^{46}$, O. Kittel ${ }^{47}$, M. Klasen ${ }^{48}$, J.-L. Kneur ${ }^{49}$, K. Kovarik ${ }^{23}$, M. Krämer ${ }^{50}$, S. Kraml ${ }^{18}$, R. Lafaye ${ }^{51}$, P. Langacker ${ }^{52}$, H.E. Logan ${ }^{53}$, W.-G. Ma ${ }^{35}$, W. Majerotto ${ }^{23}$, H.-U. Martyn ${ }^{54,2}$, K.Matchev ${ }^{55}$, D.J. Miller ${ }^{56}$, M. Mondragon ${ }^{44}$, G. Moortgat-Pick ${ }^{18}$, S. Moretti ${ }^{46}$, T. Mori ${ }^{57}$, G. Moultaka ${ }^{49}$, S. Muanza $^{58}$, M.M. Mühlleitner ${ }^{12}$, B. Mukhopadhyaya ${ }^{59}$, U. Nauenberg ${ }^{60}$, M.M. Nojiri ${ }^{61}$, D. Nomura ${ }^{13}$, H. Nowak ${ }^{62}$, N. Okada ${ }^{34}$, K.A. Olive ${ }^{63}$, W. Öller ${ }^{23}$, M. Peskin ${ }^{11}$, T. Plehn ${ }^{27}$, G. Polesello ${ }^{64}$, W. Porod ${ }^{39,26 e}$, F. Quevedo ${ }^{3}$, D. Rainwater ${ }^{65}$, J. Reuter ${ }^{2}$, P. Richardson ${ }^{66}$, K. Rolbiecki $^{42} d$, P. Roy ${ }^{67}$, R. Rückl ${ }^{25}$, H. Rzehak ${ }^{68}$, P. Schleper ${ }^{69}$, K. Siyeon ${ }^{70}$, P. Skands ${ }^{16}$, P. Slavich ${ }^{12}$, D. Stöckinger ${ }^{66}$, P. Sphicas ${ }^{18}$, M. Spira ${ }^{68}$, T. Tait ${ }^{7}$, D.R. Tovey ${ }^{71}$, J.W.F. Valle ${ }^{39}$, C.E.M. Wagner ${ }^{72,7}$, Ch. Weber ${ }^{23}$, G. Weiglein ${ }^{66}$, P. Wienemann ${ }^{19}$, Z.-Z. Xing ${ }^{73}, Y$. Yamada ${ }^{74}$, J.M. Yang ${ }^{73}$, D. Zerwas ${ }^{21}$, P.M. Zerwas ${ }^{2}$, R.-Y. Zhang ${ }^{35}$, X. Zhang ${ }^{73}$, S.-H. Zhu ${ }^{75}$
\end{abstract}

${ }^{1}$ Departamento de Fisica and CFTP, Instituto Superior Tecnico, Lisbon, Portugal

${ }^{2}$ Deutsches Elektronen-Synchrotron DESY, Hamburg, Germany

${ }^{3}$ DAMTP, University of Cambridge, Cambridge, UK

${ }_{5}^{4}$ Department of Physics, Texas A\&M University, College Station, TX, USA

${ }^{5}$ Department of Physics, Florida State University, Tallahassee, FL, USA

${ }^{6}$ Department of Physics and Astronomy, Johns Hopkins University, Baltimore, MD, USA

${ }^{7}$ High Energy Physics Division, Argonne National Laboratory, Argonne, IL, USA

8 Department of Physics, University of Wisconsin, Madison, WI, USA

${ }^{9}$ Lawrence Berkeley National Laboratory, Berkeley, CA, USA

10 Institut für Theoretische Physik, Universität Wien, Wien, Austria

11 Stanford Linear Accelerator Center, Stanford, CA, USA

12 Laboratoire de Physique Theorique, Annecy-le-Vieux, France

${ }^{13}$ Department of Physics and Astronomy, Michigan State University, East Lansing, MI, USA

${ }^{14}$ Royal Holloway University of London, Egham, Surrey, UK

15 Skobeltsyn Institute of Nuclear Physics, MSU, Moscow, Russia

${ }^{16}$ Fermi National Accelerator Laboratory, Batavia, IL, USA

17 Department of Physics, Chonbuk National University, Chonju, Korea

18 PH Department, CERN, Geneva, Switzerland

19 Physikalisches Institut, Universität Freiburg, Freiburg, Germany

${ }^{20}$ Physics Department, Universidad Catolica de Chile, Santiago, Chile

${ }^{21}$ LAL, Université de Paris-Sud, IN2P3-CNRS, Orsay, France

${ }^{22}$ University of Delhi, Delhi, India

${ }^{23}$ Institut für Hochenergiephysik, Österreichische Akademie der Wissenschaften, Wien, Austria

${ }^{24}$ Instituto de Física, UNAM, México, Mexico

${ }^{25}$ Institut für Theoretische Physik und Astrophysik, Universität Würzburg, Würzburg, Germany

${ }^{26}$ Institut für Theoretische Physik, Universität Zürich, Zürich, Switzerland

27 Max-Planck-Institut für Physik, München, Germany

${ }^{28}$ Centre for High Energy Physics, Indian Institute of Science, Bangalore, India

${ }^{29}$ Department of Theoretical Physics, Aristotle University of Thessaloniki, Thessaloniki, Greece

${ }^{30}$ Facultat de Física, Universitat de Barcelona, Barcelona, Spain

31 Department of Physics, University of California, Davis, CA, USA

32 Institute of Theoretical Physics, University of Tokushima, Tokushima, Japan

33 Santa Cruz Institute for Particle Physics, University of California, Santa Cruz, CA, USA

34 Theory Division, KEK, Tsukuba, Japan 
${ }^{35}$ Department of Modern Physics, University of Science and Technology of China, Hefei, China

${ }^{36}$ Center for High Energy Physics and Institute of Modern Physics, Tsinghua University, Beijing, China

37 High Energy Physics, Uppsala University, Uppsala, Sweden

38 Department of Physics, Tokyo Gakugei University, Tokyo, Jpan

39 Instituto de Física Corpuscular, CSIC, València, Spain

40 Department of Physics, National Taiwan University, Taipei, Taiwan

41 Department of Mathematical Sciences, University of Liverpool, Liverpool, UK

42 Institute of Theoretical Physics, Warsaw Univerity, Warsaw, Poland

${ }^{43}$ MCTP, University of Michigan, Ann Arbor, MI, USA

44 School of Physics, Seoul National University, Seoul, Korea

45 Department of Physics, Yonsei University, Seoul, Korea

46 School of Physics and Astronomy, University of Southampton, Southampton, UK

47 Physikalisches Institut der Universität Bonn, Bonn, Germany

48 Laboratoire de Physique Subatomique et de Cosmologie, Université Grenoble I, Grenoble, France

49 LPTA, Université Montpellier II, CNRS-IN2P3, Montpellier, France

${ }^{50}$ Institut für Theoretische Physik, RWTH Aachen, Aachen, Germany

${ }^{51}$ Laboratoire de Physique des Particules, Annecy-le-Vieux, France

52 Department of Physics and Astronomy, University of Pennsylvania, Philadelphia, PA, USA

53 Department of Physics, Carleton University, Ottawa, ON, Canada

54 I. Physikalisches Institut der RWTH Aachen, Aachen, Germany

55 Department of Physics, University of Florida, Gainesville, FL, USA

56 Department of Physics and Astronomy, University of Glasgow, Glasgow, UK

57 ICEPP, University of Tokyo, Tokyo, Japan

${ }^{58}$ IPN Université Lyon, IN2P3-CNRS, Lyon, France

59 Harish-Chandra Research Institute, Allahabad, India

60 University of Colorado, Boulder, CO, USA

61 YITP, Kyoto Universty, Kyoto, Japan

62 Deutsches Elektronen-Synchrotron DESY, Zeuthen, Germany

63 William I. Fine Theoretical Physics Institute, University of Minnesota, Minneapolis, MN, USA

${ }^{64}$ INFN, Sezione di Pavia, Pavia, Italy

65 Department of Physics and Astronomy, University of Rochester, Rochester, NY, USA

${ }^{66}$ IPPP, University of Durham, Durham, UK

${ }^{67}$ Tata Institute of Fundamental Research, Mumbai, India

${ }^{6}$ Paul Scherrer Institut, Villigen, Switzerland

69 Institut für Experimentalphysik, Universität Hamburg, Hamburg, Germany

70 Department of Physics, Chung-Ang University, Seoul, Korea

71 Department of Physics and Astronomy, University of Sheffield, Sheffield, UK

72 Enrico Fermi Institute, University of Chicago, Chicago, IL, USA

73 Institute of High Energy Physics, Chinese Academy of Sciences, Beijing, China

${ }^{74}$ Department of Physics, Tohoku University, Sendai, Japan

75 ITP, School of Physics, Peking University, Beijing, China

${ }^{a}$ Supported in part by US DOE, Div. of HEP, contract W-31-109-ENG-38

${ }^{b}$ Supported in part by UNAM grant PAPIIT-IN116202 and Conacyt grant 42026-F

${ }^{c}$ Heisenberg Fellow

${ }^{d}$ Supported by grant KBN 2 P03B 04024

e Supported by a MCyT Ramon y Cajal contract

January 29, 2007

Abstract. High-precision analyses of supersymmetry parameters aim at reconstructing the fundamental supersymmetric theory and its breaking mechanism. A well defined theoretical framework is needed when higher-order corrections are included. We propose such a scheme, Supersymmetry Parameter Analysis SPA, based on a consistent set of conventions and input parameters. A repository for computer programs is provided which connect parameters in different schemes and relate the Lagrangian parameters to physical observables at LHC and high energy $e^{+} e^{-}$linear collider experiments, i.e., masses, mixings, decay widths and production cross sections for supersymmetric particles. In addition, programs for calculating high-precision low energy observables, the density of cold dark matter (CDM) in the universe as well as the cross sections for CDM search experiments are included. The SPA scheme still requires extended efforts on both the theoretical and experimental side before data can be evaluated in the future at the level of the desired precision. We take here an initial step of testing the SPA scheme by applying the techniques involved to a specific supersymmetry reference point. 


\section{INTRODUCTION}

At future colliders, experiments can be performed in the supersymmetric particle sector [1,2,3, 4, if realized in Nature, with very high precision. While the Large Hadron Collider LHC can provide us with a set of welldetermined observables 56, in particular masses of colored particles and precise mass differences of various particle combinations, experiments at the International $e^{+} e^{-}$Linear Collider ILC 7, 8,9] offer highprecision determination of the non-colored supersymmetry sector. Combining the information from LHC on the generally heavy colored particles with the information from ILC on the generally lighter non-colored particle sector (and later from the Compact Linear Collider CLIC [10] on heavier states) will generate a comprehensive high-precision picture of supersymmetry at the TeV scale 11. Such an analysis can be performed independently of specific model assumptions and for any supersymmetric scenario that can be tested in laboratory experiments. It may subsequently serve as a solid base for the reconstruction of the fundamental supersymmetric theory at a high scale, potentially close to the Planck scale, and for the analysis of the microscopic mechanism of supersymmetry breaking [12,13].

The analyses will be based on experimental accuracies expected at the percent down to the per-mil level 9.14. These experimental accuracies must be matched on the theoretical side. This demands a well-defined framework for the calculational schemes in perturbation theory as well as for the input parameters. The proposed Supersymmetry Parameter Analysis Convention (SPA) [Sect.2] provides a clear base for calculating masses, mixings, decay widths and production cross sections. They will serve to extract the fundamental supersymmetric Lagrangian parameters and the supersymmetry-breaking parameters from future data. In addition, the renormalization group techniques must be developed for all the scenarios to determine the high-scale parameters of the supersymmetric theory and its microscopic breaking mechanism.

By constructing such a coherent and unified basis, the comparison between results from different calculations can be streamlined, eliminating ambiguous procedures and reducing confusion to a minimum when cross-checking results.

A program repository [Sect.3] has therefore been built in which a series of programs has been collected that will be expanded continuously in the future. The programs relate parameters defined in different schemes with each other, e.g. pole masses with $\overline{\mathrm{DR}}$ masses, and they calculate decay widths and cross sections from the basic Lagrangian parameters. An additional set of programs predicts the values of high-precision low-energy observables of Standard Model (SM) particles in supersymmetric theories. The program repository also includes global fit programs by which the entire set of Lagrangian parameters, incorporating higher-order corrections, can be extracted from the experimental observables. In addition, the solutions of the renormalization group equations are included by which extrapolations from the laboratory energies to the Grand Unification (GUT) and Planck scales can be performed and vice versa. Another category contains programs which relate the supersymmetry (SUSY) parameters with the predictions of cold dark matter in the universe and the corresponding cross sections for search experiments of cold dark matter (CDM) particles.

It is strongly recommended that the programs available in the repository adopt the structure of Ref. 15] for the Lagrangian, including flavor mixing and $\mathrm{CP}$ phases, and follow the generally accepted Supersymmetry Les Houches Accord, SLHA, for communication between different programs [16]. For definiteness, we reproduce from [16] the superpotential (omitting $R$ parity violating terms), in terms of superfields,

$$
\begin{aligned}
W=\epsilon_{a b} & {\left[\left(Y_{E}\right)_{i j} \hat{H}_{d}^{a} \hat{L}_{i}^{b} \hat{\bar{E}}_{j}+\left(Y_{D}\right)_{i j} \hat{H}_{d}^{a} \hat{Q}_{i}^{b} \hat{\bar{D}}_{j}\right.} \\
& \left.+\left(Y_{U}\right)_{i j} \hat{H}_{u}^{b} \hat{Q}_{i}^{a} \hat{\bar{U}}_{j}-\mu \hat{H}_{d}^{a} \hat{H}_{u}^{b}\right],
\end{aligned}
$$

where the chiral superfields of the Minimal Supersymmetric Standard Model (MSSM) have the following $S U(3)_{C} \otimes S U(2)_{L} \otimes U(1)_{Y}$ quantum numbers

$$
\begin{aligned}
& \hat{L}:\left(1,2,-\frac{1}{2}\right), \hat{\bar{E}}:(1,1,1), \hat{Q}:\left(3,2, \frac{1}{6}\right), \hat{\bar{U}}:\left(\overline{3}, 1,-\frac{2}{3}\right) \\
& \hat{\bar{D}}:\left(\overline{3}, 1, \frac{1}{3}\right), \hat{H}_{d}:\left(1,2,-\frac{1}{2}\right), \hat{H}_{u}:\left(1,2, \frac{1}{2}\right) .
\end{aligned}
$$

The indices of the $S U(2)_{L}$ fundamental representation are denoted by $a, b=1,2$ and the generation indices by $i, j=1,2,3$. Color indices are everywhere suppressed, since only trivial contractions are involved. $\epsilon_{a b}$ is the totally antisymmetric tensor, with $\epsilon_{12}=\epsilon^{12}=1$.

The soft SUSY breaking part is written as

$$
\begin{aligned}
& -\mathcal{L}_{s o f t}=\epsilon_{a b}\left[\left(T_{E}\right)_{i j} H_{d}^{a} \tilde{L}_{i_{L}}^{b} \tilde{e}_{j_{R}}^{*}+\left(T_{D}\right)_{i j} H_{d}^{a} \tilde{Q}_{i_{L}}^{b} \tilde{d}_{j_{R}}^{*}\right. \\
& \left.\quad+\left(T_{U}\right)_{i j} H_{u}^{b} \tilde{Q}_{i_{L}}^{a} \tilde{u}_{j_{R}}^{*}\right]+ \text { h.c. } \\
& +m_{H_{d}}^{2} H_{d a}^{*} H_{d}^{a}+m_{H_{u}}^{2} H_{u a}^{*} H_{u}^{a}-\left(m_{3}^{2} \epsilon_{a b} H_{d}^{a} H_{u}^{b}+\text { h.c. }\right) \\
& +\tilde{Q}_{i_{L} a}^{*}\left(m_{\tilde{Q}}^{2}\right)_{i j} \tilde{Q}_{j_{L}}^{a}+\tilde{L}_{i_{L} a}^{*}\left(m_{\tilde{L}}^{2}\right)_{i j} \tilde{L}_{j_{L}}^{a} \\
& +\tilde{u}_{i_{R}}\left(m_{\tilde{u}}^{2}\right)_{i j} \tilde{u}_{j_{R}}^{*}+\tilde{d}_{i_{R}}\left(m_{\tilde{d}}^{2}\right)_{i j} \tilde{d}_{j_{R}}^{*}+\tilde{e}_{i_{R}}\left(m_{\tilde{e}}^{2}\right)_{i j} \tilde{e}_{j_{R}}^{*} \\
& +\frac{1}{2}\left(M_{1} \tilde{b} \tilde{b}+M_{2} \tilde{w}^{A} \tilde{w}^{A}+M_{3} \tilde{g}^{X} \tilde{g}^{X}\right)+\text { h.c. }
\end{aligned}
$$

where the $H_{i}$ are the scalar Higgs fields, the fields with a tilde are the scalar components of the superfield with the identical capital letter; the bino is denoted as $\tilde{b}$, the unbroken $S U(2)_{L}$ gauginos as $\tilde{w}^{A=1,2,3}$, and the gluinos as $\tilde{g}^{X=1 \ldots 8}$, in 2-component notation. The $T$ matrices will be decomposed as $T_{i j}=A_{i j} Y_{i j}$, where $Y$ are the Yukawa matrices and $A$ the soft supersymmetry breaking trilinear couplings.

Much work on both the theoretical and the experimental side is still needed before data could be evaluated in the future at the desired level of accuracy. 


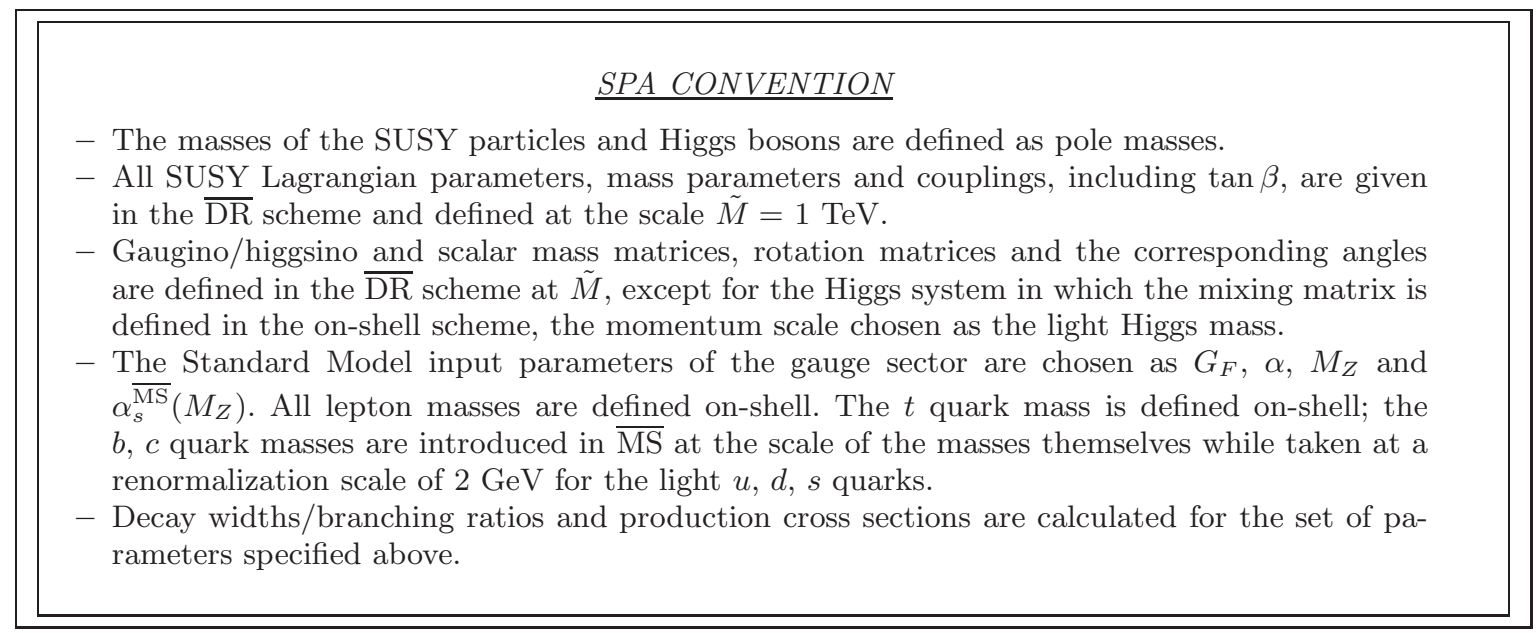

Table 1. Definition of the supersymmetry parameter convention SPA

These tasks of the SPA Project will be defined in detail in Sect.4.

In Sect.5 we introduce the SUSY reference point SPS1a' as a general setup for testing these tools in practice. This reference point is defined at a characteristic scale of $1 \mathrm{TeV}$ in the Minimal Supersymmetric Standard Model with roots in minimal supergravity (mSUGRA). The point is a derivative of the Snowmass point SPS1a [17; its parameters are identical except for a small shift of the scalar mass parameter and a change of the trilinear coupling to comply with the measured dark matter density 18. Note, that the SPS1a' parameters are compatible with all the available high- and low-energy data. The parameters are close to point $\mathrm{B}^{\prime}$ of Ref. [19. The masses are fairly light so that stringent tests of all aspects in the program can be performed for LHC and ILC experiments. The final target are predictions on the accuracies of the fundamental supersymmetry parameters that can be expected from a common set of information when LHC and ILC experiments are analyzed coherently.

Additional benchmark points within and beyond mSUGRA, representing characteristics of different scenarios, should complement the specific choice of SPS1a'.

\section{SPA CONVENTION}

Extending the experience collected in analyzing Standard Model parameters at the former $e^{+} e^{-}$colliders LEP and SLC, we propose the set of conventions defined in Table 1. These conventions conform with the general SLHA scheme 16 but they are more specific in several points.

Though largely accepted as standard, some of the definitions proposed in this SPA Convention should be explained in a few comments.

For the SUSY Lagrangian parameters the $\overline{\mathrm{DR}}$ scheme 2021 is most useful. It is based on regularization by dimensional reduction together with modified minimal subtraction. This scheme is designed to preserve supersymmetry by maintaining the number of degrees of freedom of all fields in $D$ dimensions, and it is technically very convenient. The $\beta$-functions for SUSY parameters in this scheme are known up to 3-loop order 22 . It has recently been shown 23 that inconsistencies of the original scheme 24] can be overcome and that the $\overline{\mathrm{DR}}$ scheme can be formulated in a mathematically consistent way. The ambiguities associated with the treatment of the Levi-Civita tensor can be parameterized as renormalization scheme dependence as was argued in 25. Checks by explicit evaluation of the supersymmetric Slavnov-Taylor identities at the one-loop level have shown that the $\overline{\mathrm{DR}}$ method generates the correct counter terms [26]. [We will use the version of the $\overline{\mathrm{DR}}$ scheme as given in 21, there referred to as $\overline{\mathrm{DR}}^{\prime}$ scheme.] To make use of the highly developed infrastructure for proton colliders, which is based on the $\overline{\mathrm{MS}}$ factorization scheme 27 , a dictionary is given in Sect.3.2 for the translation between the $\overline{\mathrm{DR}}$ and $\overline{\mathrm{MS}}$ schemes, as well as the on-shell renormalization schemes.

The SUSY scale is chosen $\tilde{M}=1 \mathrm{TeV}$ to avoid large threshold corrections in running the mass parameters by renormalization group techniques from the high scale down to the low scale. Fixing the scale $\tilde{M}$ independent of parameters within the supersymmetry scenarios is preferable over choices relating to specific parameters, such as squark masses, that can be fixed only at the very end. By definition, this point can also be used to characterize uniquely multiple-scale approaches.

Mixing parameters, in particular $\tan \beta$, could have been introduced in different ways [29]; however, choosing the $\overline{\mathrm{DR}}$ definitions proposed above has proven very convenient in practical calculations.

The masses of Higgs bosons 30, in the MSSM of the charged $H^{ \pm}$, of the neutral $C P$-odd $A$, and of the two $C P$-even $h, H$ particles, are understood as pole masses, $M_{H^{ \pm}, A, H, h}$. For given $M_{A}$, the pole masses $M_{H, h}$ of the $C P$-even Higgs bosons are obtained as 
poles $q^{2}=M_{H, h}^{2}$ of the dressed propagator matrix,

$\Delta_{\mathrm{Hh}}\left(q^{2}\right)=\left(\begin{array}{cc}q^{2}-m_{H}^{2}+\Sigma_{H H}\left(q^{2}\right) & \Sigma_{h H}\left(q^{2}\right) \\ \Sigma_{h H}\left(q^{2}\right) & q^{2}-m_{h}^{2}+\Sigma_{h h}\left(q^{2}\right)\end{array}\right)^{-1}$

involving the tree-level masses $m_{H, h}$ and the diagonal and non-diagonal on-shell-renormalized self-energies $\Sigma$. In the on-shell scheme, the input parameters are renormalized on-shell quantities, in particular the $A$-boson mass, with accordingly defined counter terms.

Owing to the momentum dependence of the selfenergies, there is no unique mixing angle $(\alpha)$ for the neutral $C P$-even Higgs system beyond the tree level, and the SPA choice can be understood as a convention for an "improved Born approximation". A convenient choice for $q^{2}$ in the self-energies which minimizes the difference of such an approximation with respect to calculations involving the proper self-energies in physical matrix elements, is given by $q^{2}=M_{h}^{2}$.

The physical on-shell masses are introduced in the decay widths and production cross sections such that the phase space is treated in the observables closest to experimental on-shell kinematics. This applies to the heavy particles while the masses of the light particles can generally be neglected in high energy processes.

In the chargino/neutralino sector the number of observable masses exceeds the number of free parameters in the system, gaugino/higgsino mass parameters and $\tan \beta$. The most convenient set of input chargino/neutralino masses is dictated by experiment [the three lowest mass states in this sector, for example] while the additional masses are subsequently predicted uniquely. Similar procedures need to be followed in the sfermion sector.

\section{PROGRAM BASE}

\subsection{PROGRAM CATEGORIES}

The computational tasks that are involved in the SPA Project can be broken down to several categories. Each of the codes that will be collected in the SPA program repository is included in one or more of these categories. It is understood that in each case the theoretical state-of-the-art precision is implemented. For communication between codes SLHA [16] is strongly recommended, which is extended in a suitable way where appropriate.

1) Scheme translation tools:

The communication between codes that employ different calculational schemes requires a set of translation rules. In the SPA program repository we therefore collect tools that implement, in particular, the definitions and relations between on-shell, $\overline{\mathrm{DR}}$ and $\overline{\mathrm{MS}}$ parameters in the Lagrangian as listed in Sect. 3.2 below.

2) Spectrum calculators:

This category includes codes of the transition from the Lagrangian parameters to a basis of physical particle masses and the related mixing matrices. This task mainly consists of deriving the on-shell particle masses (including higher-order corrections) and of diagonalizing the mixing matrices in a consistent scheme, making use of the abovementioned tools as needed.

3) Calculation of other observables:

3 A) Decay tables:

compute the experimentally measurable widths and branching fractions.

3B) Cross sections:

calculate SUSY cross sections and distributions for LHC and ILC.

3C) Low-energy observables: compute the values of those low-energy, highprecision observables $\left[e . g ., b \rightarrow s \gamma, B_{s} \rightarrow \mu \mu\right.$, $\left.g_{\mu}-2\right]$ that are sensitive to SUSY effects.

3D) Cosmological and astrophysical aspects: this category of programs covers the derivation of cold dark matter (CDM) relic density in the universe, cross sections for CDM particle searches, astrophysical cross sections, etc. in the SUSY context.

4) Event generators:

Programs that generate event samples for SUSY and background processes in realistic collider environments.

5) Analysis programs:

These codes make use of some or all of the above to extract the Lagrangian parameters from experimental data by means of global analyses.

6) RGE programs:

By solving the renormalization-group equations, the programs connect the values of the parameters of the low-energy effective Lagrangian to those at the high-scale where the model is supposed to match to a more fundamental theory. High-scale constraints are implemented on the basis of well-defined theoretical assumptions: gauge coupling unification, mSUGRA, GMSB, AMSB scenarios, etc.

7) Auxiliary programs and libraries:

Structure functions, beamstrahlung, numerical methods, SM backgrounds, etc.

This is an open system and the responsibility for all these programs remains with the authors. SPA provides the translation tables and the links to the computer codes on the web-page

$$
\text { http://spa.desy.de/spa/ }
$$

Conveners responsible for specific tasks of the SPA Project will be listed on this web-page; the information will be routinely updated to reflect the momentary state of the project at any time.

\subsection{SCHEME TRANSLATION}

This subsection presents a few characteristic examples of relations between on-shell observables and $\overline{\mathrm{DR}}, \overline{\mathrm{MS}}$ quantities at the electroweak scale $M_{Z}$ and the SUSY 
scale $\tilde{M}$. For brevity, here only the approximate oneloop results are given [31; it is understood that the codes in the program repository include the most upto-date higher-loop results.

(a) Couplings:

- gauge couplings:

$$
g_{i}^{\overline{\mathrm{MS}}}=g_{i}^{\overline{\mathrm{DR}}}\left(1-\frac{\left(g_{i}^{\overline{\mathrm{DR}}}\right)^{2}}{96 \pi^{2}} C_{i}\right)
$$

- Yukawa couplings between the gaugino $\lambda_{i}$, the chiral fermion $\psi_{k}$ and the scalar $\phi_{k}$ :

$$
\hat{g}_{i k}^{\overline{\mathrm{MS}}}=g_{i}^{\overline{\mathrm{DR}}}\left(1+\frac{\left(g_{i}^{\overline{\mathrm{DR}}}\right)^{2}}{32 \pi^{2}} C_{i}-\sum_{l=1}^{3} \frac{\left(g_{l}^{\overline{\mathrm{DR}}}\right)^{2}}{32 \pi^{2}} C_{l}^{r_{k}}\right)
$$

- Yukawa couplings between the scalar $\phi_{i}$ and the two chiral fermions $\psi_{j}$ and $\psi_{k}$ :

$$
Y_{i j k}^{\overline{\mathrm{MS}}}=Y_{i j k}^{\overline{\mathrm{DR}}}\left(1+\sum_{l=1}^{3} \frac{\left(g_{l}^{\overline{\mathrm{DR}}}\right)^{2}}{32 \pi^{2}}\left[C_{l}^{r_{j}}-2 C_{l}^{r_{i}}+C_{l}^{r_{k}}\right]\right)
$$

- trilinear scalar couplings:

These couplings do not differ in the two schemes.

$C_{i}$ and $C_{i}^{r}$ are the quadratic Casimir invariants of the adjoint representation and the matter representation $r$ of the gauge group $G_{i}$, respectively. They are given by $C_{i}=[3,2,0]$ for $[S U(3), S U(2), U(1)]$ and $C_{i}^{r}=\left[4 / 3,3 / 4,3 / 5 \times Y_{r}^{2}\right]$ for the fundamental representations of $S U(3), S U(2)$, and the $U(1)$ hypercharge $Y_{r}$.

(b) SUSY $\overline{\mathrm{DR}}, \overline{\mathrm{MS}}$ and pole masses:

- gaugino mass parameters

$$
M_{i}^{\overline{\mathrm{MS}}}=M_{i}^{\overline{\mathrm{DR}}}\left(1+\frac{\left(g_{i}^{\overline{\mathrm{DR}}}\right)^{2}}{16 \pi^{2}} C_{i}\right)
$$

- higgsino mass parameter:

$$
\mu^{\overline{\mathrm{MS}}}=\mu^{\overline{\mathrm{DR}}}\left(1+\sum_{l=1}^{2} \frac{\left(g_{l}^{\overline{\mathrm{DR}}}\right)^{2}}{16 \pi^{2}} C_{l}^{H}\right)
$$

$C_{l}^{H}$ denoting the $S U(2)$ and $U(1)$ Casimir invariants of the Higgs fields.

\section{- sfermion mass parameters:}

These parameters do not differ in the $\overline{\mathrm{DR}}$ and $\overline{\mathrm{MS}}$ schemes.

- fermion pole masses:

The pole masses can be written schematically as

$$
m_{i, \text { pole }}=M_{i}^{\overline{\mathrm{DR}}}-\operatorname{Re} \Sigma\left(\not=m_{i, \text { pole }}\right)
$$

where $\Sigma$ denotes the fermion self-energy renormalized according to the $\overline{\mathrm{DR}}$-scheme at the scale $\tilde{M}$. As an explicit example we note the one-loop relation between the $\mathrm{SU}(3)$ gaugino mass parameter $M_{3}(\tilde{M})^{\overline{\mathrm{DR}}}$ and the gluino pole mass $m_{\tilde{g}}$ [without sfermion mixing] at the one-loop order:

$$
\begin{aligned}
m_{\tilde{g}}= & M_{3}^{\overline{\mathrm{DR}}}(\tilde{M}) \\
+\frac{\alpha_{s}^{\overline{\mathrm{DR}}}(\tilde{M})}{4 \pi}[ & {\left[m_{\tilde{g}}\left(15+9 \ln \frac{\tilde{M}^{2}}{m_{\tilde{g}}^{2}}\right)\right.} \\
& \left.+\sum_{q} \sum_{i=1}^{2} m_{\tilde{g}} B_{1}\left(m_{\tilde{g}}^{2}, m_{q}^{2}, m_{\tilde{q}_{i}}^{2}\right)\right]
\end{aligned}
$$

where $B_{1}$ is the finite part of one of the one-loop two-point functions at the scale in the $\overline{\mathrm{DR}}$ scheme $\tilde{M}$ (and analogously $A_{0}, B_{0}$ to be used later), cf. Ref. 32.

- scalar pole masses:

A similar relation holds for the squared scalar masses

$$
m_{i, \text { pole }}^{2}=M_{i}^{2, \overline{\mathrm{DR}}}-\Sigma\left(q^{2}=m_{i, \text { pole }}^{2}\right)
$$

The one-loop QCD corrections for the left squarks of the first two generations in the limit of vanishing quark masses may serve as a simple example:

$$
\begin{aligned}
m_{\tilde{q}}^{2}= & M_{\tilde{Q}}^{2, \overline{\mathrm{DR}}}(\tilde{M}) \\
& -\frac{2 \alpha_{s}^{\overline{\mathrm{DR}}}(\tilde{M})}{3 \pi}\left[\left(m_{\tilde{q}}^{2}-m_{\tilde{g}}^{2}\right) B_{0}\left(m_{\tilde{q}}^{2}, m_{\tilde{g}}^{2}, 0\right)\right. \\
& \left.-2 m_{\tilde{q}}^{2} B_{0}\left(m_{\tilde{q}}^{2}, m_{\tilde{q}}^{2}, 0\right)+A_{0}\left(m_{\tilde{q}}^{2}\right)-A_{0}\left(m_{\tilde{g}}^{2}\right)\right]
\end{aligned}
$$

(c) SM parameters:

The following paragraphs summarize the SM input values for the analysis. Only approximate formulae are presented for brevity, while the complete set of relations is available on the program repository. In a few cases the evolution from the scale $M_{Z}$ to $\tilde{M}$ is carried out by means of RGEs instead of fixed-order perturbation theory because they have proven, presently, more accurate; this may change once the necessary multi-loop calculations will be completed.

- $\alpha, \alpha^{\overline{\mathrm{DR}}}\left(M_{Z}\right), \alpha_{1,2}^{\overline{\mathrm{DR}}}(\tilde{M})$ :

$$
\begin{aligned}
& \alpha^{\overline{\mathrm{DR}}}\left(M_{Z}\right)= \frac{\alpha}{1-\Delta \alpha_{\mathrm{SM}}-\Delta \alpha_{\mathrm{SUSY}}} \\
& \Delta \alpha_{\mathrm{SUSY}}=-\frac{\alpha}{6 \pi}\left[\ln \frac{m_{H^{+}}}{M_{Z}}+4 \sum_{i=1}^{2} \ln \frac{m_{\tilde{\chi}_{i}^{+}}}{M_{Z}}\right. \\
&\left.+\sum_{f} \sum_{i=1}^{2} N_{c} Q_{f}^{2} \ln \frac{m_{\tilde{f}_{i}}}{M_{Z}}\right]
\end{aligned}
$$


$\Delta \alpha_{\mathrm{SM}}$ summarizes the SM contributions from the leptons, quarks and the $W$-boson. In the SUSY contributions, $\Delta \alpha_{\text {SUSY }}, f$ sums over all charged sfermions, $N_{c}$ is the color factor and $Q_{f}$ the (s)fermion charge.

$$
\begin{aligned}
& \alpha_{1}^{\overline{\mathrm{DR}}}(\tilde{M})= \frac{\frac{\alpha^{\overline{\mathrm{DR}}}\left(M_{Z}\right)}{1+\frac{1}{4 \pi} \frac{\alpha^{2} \theta^{\overline{\mathrm{DR}}}}{\cos ^{2} \theta^{\overline{\mathrm{DR}}}}\left(M_{Z}\right)}}{\ln \frac{M_{Z}^{2}}{\tilde{M}^{2}}} \\
& \alpha_{2}^{\overline{\mathrm{DR}}}(\tilde{M})=\frac{\frac{\alpha^{\overline{\mathrm{DR}}}\left(M_{Z}\right)}{\sin ^{2} \theta^{\overline{\mathrm{DR}}}\left(M_{Z}\right)}}{1+\frac{1}{4 \pi} \frac{\alpha^{\overline{\mathrm{DR}}}\left(M_{Z}\right)}{\sin ^{2} \theta^{\overline{\mathrm{DR}}}\left(M_{Z}\right)} \ln \frac{M_{Z}^{2}}{\tilde{M}^{2}}}
\end{aligned}
$$

- $\sin ^{2} \theta^{\overline{\mathrm{DR}}}$ at $M_{Z}$ and at $\tilde{M}$ :

The electroweak mixing parameter $\sin ^{2} \theta^{\overline{\mathrm{DR}}}\left(M_{Z}\right)$ is given by

$$
\begin{array}{r}
\sin ^{2} \theta^{\overline{\mathrm{DR}}}\left(M_{Z}\right)\left[1-\sin ^{2} \theta^{\overline{\mathrm{DR}}}\left(M_{Z}\right)\right] \\
=\frac{\pi \alpha^{\overline{\mathrm{DR}}}\left(M_{Z}\right)}{\sqrt{2} M_{Z}^{2} G_{F}(1-\Delta \hat{r})}
\end{array}
$$

where the contributions from loops of SM and SUSY particles are denoted by $\Delta \hat{r} 33,34$. At the scale $\tilde{M}$ the electroweak mixing parameter can be calculated subsequently from

$$
\tan ^{2} \theta^{\overline{\mathrm{DR}}}(\tilde{M})=\alpha_{1}^{\overline{\mathrm{DR}}}(\tilde{M}) / \alpha_{2}^{\overline{\mathrm{DR}}}(\tilde{M})
$$

by making use of the couplings $\alpha_{i}^{\overline{\mathrm{DR}}}(\tilde{M})$ given in the preceeding paragraph.

- $\sin ^{2} \theta^{\overline{\mathrm{DR}}}$ and $\sin ^{2} \theta_{\text {eff }}$ at $M_{Z}$ :

The electroweak mixing angle in the effective leptonic (electronic) vertex of the $Z$ boson is defined as

$$
\sin ^{2} \theta_{\mathrm{eff}} \equiv \sin ^{2} \theta_{\mathrm{eff}}^{(\mathrm{e})}\left(M_{Z}\right)=\frac{1}{4}\left(1-\operatorname{Re} \frac{g_{V}^{\mathrm{e}}}{g_{A}^{\mathrm{e}}}\right)
$$

in terms of the effective vector and axial vector couplings $g_{V, A}^{\mathrm{e}}$ of the $Z$ to electrons. The relation to $\sin ^{2} \theta^{\overline{\mathrm{DR}}}\left(M_{Z}\right)$ is given by (at one-loop order)

$$
\begin{aligned}
& \sin ^{2} \theta^{\overline{\mathrm{DR}}}\left(M_{Z}\right)=\sin ^{2} \theta_{\mathrm{eff}} \\
& +\sin 2 \theta_{\mathrm{eff}} \frac{\Pi_{\gamma Z}\left(M_{Z}^{2}\right)+\Pi_{\gamma Z}(0)}{2 M_{Z}^{2}}-f^{\mathrm{e}}
\end{aligned}
$$

involving the photon- $Z$ non-diagonal self-energy $\Pi_{\gamma Z}\left(q^{2}\right)$ and the non-universal electron- $Z$ vertex correction form factors $f_{V, A}^{\mathrm{e}}\left(q^{2}\right)$,

$$
f^{\mathrm{e}}=\frac{1}{2} f_{V}^{\mathrm{e}}\left(M_{Z}^{2}\right)-\left(\frac{1}{2}-2 \sin ^{2} \theta_{\mathrm{eff}}\right) f_{A}^{\mathrm{e}}\left(M_{Z}^{2}\right),
$$

with all the loop quantities renormalized in the $\overline{\mathrm{DR}}$ scheme at the scale $M_{Z}$. For explicit expressions see 33 34.

- $\alpha_{s}^{\overline{\mathrm{DR}}}$ at $M_{Z}$ and $\tilde{M}$, related to $\alpha_{s}^{\overline{\mathrm{MS}}}\left(M_{Z}\right)$ :

$$
\begin{aligned}
\alpha_{s}^{\overline{\mathrm{DR}}}\left(M_{Z}\right)= & \frac{\alpha_{s}^{\overline{\mathrm{MS}}}\left(M_{Z}\right)}{1-\Delta \alpha_{s}} \\
\Delta \alpha_{s}= & \frac{\alpha_{s}\left(M_{Z}\right)}{2 \pi}\left[\frac{1}{2}-\frac{2}{3} \ln \frac{m_{t}}{M_{Z}}\right. \\
& \left.-2 \ln \frac{m_{\tilde{g}}}{M_{Z}}-\frac{1}{6} \sum_{\tilde{q}} \sum_{i=1}^{2} \ln \frac{m_{\tilde{q}_{i}}}{M_{Z}}\right] \\
\alpha_{s}^{\overline{\mathrm{DR}}}(\tilde{M})= & \frac{\alpha_{s}^{\overline{\mathrm{DR}}}\left(M_{Z}\right)}{1-\frac{3}{4 \pi} \alpha_{s}^{\overline{\mathrm{DR}}}\left(M_{Z}\right) \ln \frac{M_{Z}^{2}}{\tilde{M}^{2}}}
\end{aligned}
$$

- $W, Z$ bosons, pole and $\overline{\mathrm{DR}}$ masses:

The pole masses $M_{V}(V=W, Z)$ and the $\overline{\mathrm{DR}}$ masses at $M_{Z}$ are related by

$$
M_{V}^{2}=M_{V}^{2, \overline{\mathrm{DR}}}\left(M_{Z}\right)-\operatorname{Re} \Pi_{V V}^{T}\left(p^{2}=M_{V}^{2}\right)
$$

involving the renormalized transverse vector-boson self-energies in the $\overline{\mathrm{DR}}$ scheme at the scale $M_{Z}$. The $Z$ pole mass is a direct input parameter, whereas the $W$ pole mass is derived from the relation to the low-energy parameters $\alpha$ and Fermi constant $G_{F}$ according to the SPA Convention:

$$
M_{W}^{2}\left(1-\frac{M_{W}^{2}}{M_{Z}^{2}}\right)=\frac{\pi \alpha}{\sqrt{2} G_{F}(1-\Delta r)},
$$

$\Delta r$ summarizes the loop contributions from the SM and SUSY particles as given explicitly in 33 34, 35. The self-energies at the scale $\tilde{M}$ can be written symbolically as

$$
\begin{aligned}
16 \pi^{2} \Pi_{Z Z}^{T}= & 16 \pi^{2} \Pi_{Z Z, \text { SM }+ \text { Higgs }}^{T} \\
& -\sum_{f} 4 N_{c}^{f} v_{f Z, i j}^{2} \tilde{B}_{22}\left(M_{Z}^{2}, m_{\tilde{f}_{i}}^{2}, m_{\tilde{f}_{j}}^{2}\right) \\
& +\sum_{\tilde{\chi}^{0}, \tilde{\chi}^{+}}\left[f_{i j Z} H\left(M_{Z}^{2}, m_{\tilde{\chi}_{i}}, m_{\tilde{\chi}_{j}}\right)\right. \\
& \left.+2 g_{i j Z} B_{0}\left(M_{Z}^{2}, m_{\tilde{\chi}_{i}}, m_{\tilde{\chi}_{j}}\right)\right] \\
16 \pi^{2} \Pi_{W W}^{T}= & 16 \pi^{2} \Pi_{W W, \mathrm{SM}+\mathrm{Higgs}}^{T} \\
& -\sum_{f} 2 N_{c}^{f} v_{f W, i j}^{2} \tilde{B}_{22}\left(M_{W}^{2}, m_{\tilde{f}_{i}}^{2}, m_{\tilde{f}_{j}^{\prime}}^{2}\right) \\
& +\sum_{i, j}\left[f_{i j W} H\left(M_{W}^{2}, m_{\tilde{\chi}_{i}^{0}}, m_{\tilde{\chi}_{j}^{+}}\right)\right. \\
& \left.+2 g_{i j W} B_{0}\left(M_{W}^{2}, m_{\tilde{\chi}_{i}^{0}}, m_{\tilde{\chi}_{j}^{+}}\right)\right]
\end{aligned}
$$

where $v_{f V, i j}$ are the couplings of the gauge boson to sfermions and $f_{i j V}$ and $g_{i j V}$ are combinations of left- and right-couplings to charginos and neutralinos; $\tilde{B}_{22}$ and $H$ are combinations of the $B_{i}$ and $A_{i}$ 
loop functions. Detailed formulae are given in 36 .

- charm and bottom running $\overline{\mathrm{MS}}$ mass at $m_{c, b}$ and $\overline{\mathrm{DR}}$ mass at $M_{Z}$, cf. [37,38]:

$$
\begin{aligned}
& m_{b, \mathrm{SM}}^{\overline{\mathrm{DR}}}\left(M_{Z}\right)=m_{b}^{\overline{\mathrm{MS}}}\left(m_{b}\right)\left[\frac{\alpha_{s}^{\overline{\mathrm{MS}}}\left(M_{Z}\right)}{\alpha_{s}^{\overline{\mathrm{MS}}}\left(m_{b}\right)}\right]^{\frac{12}{23}} \\
& \times\left[1-\frac{\alpha_{s}^{\overline{\mathrm{DR}}}}{3 \pi}-\frac{23 \alpha_{s}^{2, \overline{\mathrm{DR}}}}{72 \pi}\right] \\
& m_{b}^{\overline{\mathrm{DR}}}\left(M_{Z}\right)=\frac{m_{b, \mathrm{SM}}^{\overline{\mathrm{DR}}}\left(M_{Z}\right)+\operatorname{Re} \Sigma_{b}^{\prime}\left(M_{Z}\right)}{1-\Delta m_{b}\left(M_{Z}\right)} \\
& \Delta m_{b}\left(M_{Z}\right)=\frac{2 \alpha_{s}}{3 \pi} m_{\tilde{g}} \mu \tan \beta I\left(m_{\tilde{b}_{1}}^{2}, m_{\tilde{b}_{2}}^{2}, m_{\tilde{g}}^{2}\right) \\
& +\frac{Y_{t}^{2}}{16 \pi^{2}} A_{t} \mu \tan \beta I\left(m_{\tilde{t}_{1}}^{2}, m_{\tilde{t}_{2}}^{2}, \mu^{2}\right) \\
& -\frac{g^{2}}{16 \pi^{2}} M_{2} \mu \tan \beta \\
& \times\left[\cos ^{2} \theta_{\tilde{t}} I\left(m_{\tilde{t}_{1}}^{2}, M_{2}^{2}, \mu^{2}\right)+\frac{1}{2}\{\tilde{t} \rightarrow \tilde{b}\}\right. \\
& \left.+\left\{\cos \rightarrow \sin ; \tilde{Q}_{1} \rightarrow \tilde{Q}_{2}\right\}\right] \\
& I\left(a^{2}, b^{2}, c^{2}\right)=\frac{a^{2} b^{2} \log a^{2} / b^{2}+\text { cyclic }}{\left(a^{2}-b^{2}\right)\left(b^{2}-c^{2}\right)\left(a^{2}-c^{2}\right)} \\
& m_{t}^{\overline{\mathrm{DR}}}\left(M_{Z}\right)=m_{t}\left[1-\frac{5 \alpha_{s}^{\overline{\mathrm{DR}}}}{3 \pi}-\frac{\alpha_{s}^{\overline{\mathrm{DR}}}}{\pi} \log \left(\frac{M_{Z}^{2}}{m_{t}^{2}}\right)\right. \\
& \left.-c_{t}\left(\frac{\alpha_{s}^{\overline{\mathrm{DR}}}}{\pi}\right)^{2}-\Sigma\right]
\end{aligned}
$$

where $c_{t}\left(M_{Z}^{2} / m_{t}^{2}\right)$ is the gluonic two-loop contribution and $\Sigma$ accounts for the electroweak as well as the SUSY contributions. The mass is evolved to the scale $\tilde{M}$ by means of the Yukawa RGEs; see next.

- Yukawa couplings and running masses of SM particles at $\tilde{M}$ :

The vacuum expectation values $v_{u}^{\overline{\mathrm{DR}}}$ and $v_{d}^{\overline{\mathrm{DR}}}$ are initially given by:

$$
M_{W}^{2}\left(M_{Z}\right)=\frac{1}{4} g^{2, \overline{\mathrm{DR}}}\left(M_{Z}\right)
$$

$$
\begin{array}{r}
\times\left[v_{u}^{2, \overline{\mathrm{DR}}}\left(M_{Z}\right)+v_{d}^{2, \overline{\mathrm{DR}}}\left(M_{Z}\right)\right] \\
v_{u}^{\overline{\mathrm{DR}}}\left(M_{Z}\right) / v_{d}^{\overline{\mathrm{DR}}}\left(M_{Z}\right)=\tan \beta^{\overline{\mathrm{DR}}}\left(M_{Z}\right)
\end{array}
$$

$\tan \beta^{\overline{\mathrm{DR}}}\left(M_{Z}\right)$ must be evolved down from the conventional parameter $\tan \beta^{\overline{\mathrm{DR}}}(\tilde{M})$ by means of RGE. From the $\overline{\mathrm{DR}}$ masses at $M_{Z}$ the Yukawa couplings are calculated:

$$
\begin{aligned}
Y_{t}^{\overline{\mathrm{DR}}}\left(M_{Z}\right) & =\sqrt{2} m_{t}^{\overline{\mathrm{DR}}}\left(M_{Z}\right) / v_{u}^{\overline{\mathrm{DR}}}\left(M_{Z}\right) \\
Y_{b, \tau}^{\overline{\mathrm{DR}}}\left(M_{Z}\right) & =\sqrt{2} m_{b, \tau}^{\overline{\mathrm{DR}}}\left(M_{Z}\right) / v_{d}^{\overline{\mathrm{DR}}}\left(M_{Z}\right)
\end{aligned}
$$

In a second step, they are evolved together with the gauge couplings and the vacuum expectation values to $\tilde{M}$ via RGEs. At this scale the running SM fermion masses and gauge boson masses are related to the Lagrangian parameters by the usual tree-level relations. This is, presently, a better approach for the evolution of the Yukawa couplings than fixed-order perturbation theory.

\subsection{WIDTHS AND CROSS SECTIONS}

(a) Decay widths:

The decay widths are defined as inclusive quantities including all radiative corrections; the masses of the heavy particles are taken on-shell, light particle masses are set zero.

(b) Cross sections for $e^{+} e^{-}$collisions:

Cross sections, $\sigma\left(e^{+} e^{-} \rightarrow\{\tilde{F}\}\right)$, for the production of a set of supersymmetric particles/Higgs bosons $\{\tilde{F}\}$ are defined at the experimental level in $e^{+} e^{-}$ collisions including up-to-date radiative corrections except hard $\gamma$ bremsstrahlung to exclude large contributions from radiative return.

In general, large QED-type photonic corrections cannot be disentangled from genuine SUSY-specific parts, and in the comparison of theoretical predictions with experimental data all higher-order terms have to be included. To elucidate the role of the specific supersymmetric loop corrections, a reasonable and consistent prescription for cut-independent reduced cross sections shall therefore be defined. Since the leading QED terms arising from virtual and real photon contributions that contain large logarithms can be identified and isolated, the "reduced" genuine SUSY cross sections are defined, at the theoretical level, by subtracting the logarithmic terms $\log 4 \Delta E^{2} / s$ in the soft-photon energy cut-off $\Delta E$ and in $\log s / m_{f}^{2}$ from non-collinear and collinear soft $\gamma$ radiation off light fermions $f=e, \mu, \ldots$ and virtual QED corrections. In this definition of reduced cross sections [see also 39], the logarithmically large QED radiative corrections are consistently eliminated in a gauge-invariant way. By the same token, the reduced cross sections are defined without taking into account beamstrahlung. 
(c) Cross sections for hadron collisions:

Cross sections for proton collisions at Tevatron and LHC, $\sigma(p p \rightarrow\{\tilde{F}\})$, include all QCD and other available corrections, with infrared and collinear singularities tamed by defining inclusive observables, or properly defined jet characteristics, and introducing the renormalized parton densities, provided parametrically by the PDF collaborations [40 41].

\section{TASKS OF THE SPA PROJECT}

A successful reconstruction of the fundamental structure of the supersymmetric theory at the high scale and the proper understanding of the nature of cold dark matter from experimental data require the precise analysis of all information that will become available from collider experiments, low-energy experiments, astrophysical and cosmological observations. Preliminary studies [see Sect.5], initiating this SPA Project, have shown that while this aim can in principle be achieved, it still needs much additional work both on the theoretical as well as on the experimental side. In particular, we identify the following areas of research as central tasks of the SPA Project:

\section{Higher-order calculations}

While the precision of SUSY calculations has gradually shifted from leading-order (LO) to next-to-leading order (NLO) accuracy [and, in some areas, beyond], the present level still does not match the expected experimental precision, particularly in coherent LHC+ILC analyses. The experimental precision, however, has to be fully exploited in order to draw firm conclusions on the fundamental theory. To close this gap, the SPA Project foresees new efforts to push the frontier in higher-order SUSY calculations to the line necessary for the proper interpretation of experimental analyses.

\section{Improving the understanding of the $\overline{\mathrm{DR}}$ scheme}

The $\overline{\mathrm{DR}}$ scheme recommended for higher-order calculations can be formulated in a mathematically consistent way [23] and is technically most convenient. Many explicit checks at the one-loop level have shown that the $\overline{\mathrm{DR}}$ method generates the correct counter terms. However, there is no complete proof yet that it preserves supersymmetry and gauge invariance in all cases. Therefore, as the precision of SUSY calculations is pushed to higher orders, the SPA Project also requires further investigation of the symmetry identities in the $\overline{\mathrm{DR}}$ scheme.

Moreover, there is an obvious dichotomy between the $\overline{\mathrm{DR}}$ scheme, which is convenient for the definition of SUSY parameters and their renormalization group evolution, and the $\overline{\mathrm{MS}}$ scheme, which is generally adopted for the calculation of hadronic processes 27. While, as argued before, the $\overline{\mathrm{MS}}$ scheme requires ad-hoc counter terms to restore supersymmetry, in the $\overline{\mathrm{DR}}$ scheme a finite shift from the commonly used $\overline{\mathrm{MS}}$ density functions to the $\overline{\mathrm{DR}}$ density functions has to be carried out 42. Moreover, for massive final state particles spurious density functions for the $(4-D)$ gluon components have to be introduced to comply with the factorization theorem, see 43 44 for details. Formulating an efficient combination of the most attractive elements of both schemes in describing hadronic processes is therefore an important task of the project.

Improving experimental and theoretical precision

The set of observables that has been included so far in experimental analyses, by no means exhausts the opportunities which data at LHC and at ILC are expected to provide in the future. SPA Project studies will aim to identify any new channels that can give additional information, either independent or redundant [improving fit results], and they will include them in a unified framework. In connection with realistic estimates of theoretical uncertainties, a solid account of error sources and correlations has to be achieved. Furthermore, the sophistication of the experimental results will be refined by including more precise signal and background calculations, and improved simulations as mandatory for the analysis of real data.

\section{Coherent LHC + ILC analyses}

We put particular emphasis on the coherent combination of future data obtained at LHC and ILC. While the LHC will most likely discover SUSY particles, if they exist, and will allow for the first tests of the SUSY paradigm, $e^{+} e^{-}$data make possible high-precision investigations of the weakly-interacting sector. Feedback and coherently combined analyses, which will greatly benefit from a concurrent running of both colliders, are indispensable for a meaningful answer to the questions raised in the present context. Studies as initiated by the LHC/LC Study Group [45] are a vital part of the SPA Project.

\section{Determining SUSY Lagrangian parameters}

While at leading order the Lagrangian parameters connected with different supersymmetric particle sectors can in general be isolated and extracted analytically from closely associated observables, the analysis is much more complex at higher orders. Higher orders introduce the interdependence of all sectors in the observables. The development of consistent analyses for the global determination of the Lagrangian parameters in this complex situation has started and, conform with general expectations for iterative steps in perturbative expansions, they can be carried out consistently with as few assumptions as possible. The set of Lagrangian parameters and their experimental error matrix can be determined, including higher-order corrections. However, the experimental procedure must still be supplemented by corresponding theoretical errors and their correlations. 


\section{Cold dark matter}

As the precision is refined, astrophysical data play an increasingly important role in confronting supersymmetry with experiments. The class of models conserving $\mathrm{R}$-parity predict a weakly interacting, massive, stable particle. The relic abundance of this particle imposes crucial limits on supersymmetric scenarios [4]. While among the supersymmetry breaking models versions of mSUGRA and of gaugino mediation 47] have been analyzed in detail, the analyses have to be extended systematically to other scenarios. In models that account for the relic density, specific requirements on the accuracies must be achieved when the CDM particle is studied in high-energy physics laboratory experiments 48. In turn, predictions based on the comprehensive parameter analysis of high-energy experiments determine the cross sections for astrophysical scattering experiments by which the nature of the cold dark matter particles can be established. The SPA Project provides a platform for a systematic and continuous interplay between the astrophysics and highenergy physics disciplines and the mutual refinement of their programs in the future.

\section{Extended SUSY scenarios}

The MSSM, in particular the parameter set SPS1a' that we suggest for a first study, provides a benchmark scenario for developing and testing the tools needed for a successful analysis of future SUSY data. However, neither this specific point nor the MSSM itself may be the correct model for low-scale SUSY. Various parameter sets [for instance other representative mSUGRA points as well as non-universal SUGRA, GMSB, AMSB, and other scenarios, see Ref. 49] for a brief summary] and extended models have therefore to be investigated within the SPA Project. In particular, models which incorporate the right-handed neutrino sector, must be analyzed extensively 50. Furthermore, $C P$ violation, $R$-parity violation, flavor violation, NMSSM and extended gauge groups are among the roads that nature may have taken in the SUSY sector. The SPA conventions are formulated so generally that they can be applied to all these scenarios. The goal of deriving the fundamental structure from data will also to be pursued for many facets in this more general context.

\section{EXAMPLE: REF POINT SPS1a'}

To test the internal consistency of the SPA scheme and to explore the potential of such extended experimental and theoretical analyses we have defined, as an example, the CP and R-parity invariant MSSM reference point SPS1a'. Of course, the SPA Convention is set up to cover also more general scenarios.

The results for SPS1a' presented below are based on preliminary experimental simulations. In some cases, however, extrapolations from earlier analyses for SPS1a and other reference points have been used in order to substitute missing information necessary for a first

\begin{tabular}{|c|c||c|c|}
\hline Parameter & SM input & Parameter & SM input \\
\hline \hline$m_{e}$ & $5.110 \cdot 10^{-4}$ & $m_{t}^{\text {pole }}$ & 172.7 \\
$m_{\mu}$ & 0.1057 & $m_{b}\left(m_{b}\right)$ & 4.2 \\
$m_{\tau}$ & 1.777 & $m_{Z}$ & 91.1876 \\
\hline$m_{u}(Q)$ & $3 \cdot 10^{-3}$ & $G_{F}$ & $1.1664 \cdot 10^{-5}$ \\
$m_{d}(Q)$ & $7 \cdot 10^{-3}$ & $1 / \alpha$ & 137.036 \\
$m_{s}(Q)$ & 0.12 & $\Delta \alpha_{h a d}^{(5)}$ & 0.02769 \\
$m_{c}\left(m_{c}\right)$ & 1.2 & $\alpha_{s}^{\overline{\mathrm{MS}}}\left(m_{Z}\right)$ & 0.119 \\
\hline
\end{tabular}

Table 2. Numerical values of the SM input to SPS1a'. Masses are given in $\mathrm{GeV}$, for the leptons and the $t$ quark the pole masses, for the lighter quarks the $\overline{\mathrm{MS}}$ masses either at the mass scale itself, for $c, b$, or, for $u, d, s$, at the scale $Q=2 \mathrm{GeV}$.

comprehensive test of all aspects of the SPA Project. It is obvious that many detailed simulations are needed to demonstrate the full power of predicting the fundamental supersymmetric parameters from future sets of LHC and ILC data.

In $e^{+} e^{-}$annihilation experimental progress is expected for the heavy chargino and neutralinos. Combining the results of such studies with LHC data appear very promising and lead to improved mass determinations 51. New techniques to determine slepton masses from cascade decays as very narrow resonances 52.53. should be applied. For cross section measurements and other sparticle properties methods to determine the decay branching ratios should be developed. At the LHC a recently proposed mass relation method offers substantial improvements in the reconstruction of squark and gluino masses 54 .

\section{Analysis of SUSY Lagrangian parameters}

The roots defining the Reference Point SPS1a' are the mSUGRA parameters [in the conventional notation for CMSSM - see [55] for the tighter original definition] in the set

$$
\begin{aligned}
& M_{1 / 2}=250 \mathrm{GeV} \quad \operatorname{sign}(\mu)=+1 \\
& M_{0}=70 \mathrm{GeV} \tan \beta(\tilde{M})=10 \\
& A_{0}=-300 \mathrm{GeV}
\end{aligned}
$$

The left column, listing the universal gaugino mass $M_{1 / 2}$, the scalar mass $M_{0}$ and the trilinear coupling $A_{0}$ [Yukawa couplings factored out], is defined at the GUT scale $M_{\mathrm{GUT}}$. The point is close to the original Snowmass point SPS1a [17; the scalar mass parameter $M_{0}$ is lowered slightly at the GUT scale from $100 \mathrm{GeV}$ to $70 \mathrm{GeV}$ and $A_{0}$ is changed from $-100 \mathrm{GeV}$ to $-300 \mathrm{GeV}$. The values of the SM input parameters are collected in Table 2 Extrapolation of the above mSUGRA parameters down to the $\tilde{M}=1 \mathrm{TeV}$ scale generates the MSSM Lagrangian parameters. Table 3 displays the couplings and mass parameters after being evolved from $M_{\mathrm{GUT}}$ to $\tilde{M}$ using the RGE part of the program SPheno [56] which is based on two-loop anal- 


\begin{tabular}{|c|c||c|c|}
\hline Parameter & SPS1a $^{\prime}$ value & Parameter & SPS1a $^{\prime}$ value \\
\hline \hline$g^{\prime}$ & 0.3636 & $M_{1}$ & 103.3 \\
$g$ & 0.6479 & $M_{2}$ & 193.2 \\
$g_{s}$ & 1.0844 & $M_{3}$ & 571.7 \\
\hline$Y_{\tau}$ & 0.1034 & $A_{\tau}$ & -445.2 \\
$Y_{t}$ & 0.8678 & $A_{t}$ & -565.1 \\
$Y_{b}$ & 0.1354 & $A_{b}$ & -943.4 \\
\hline$\mu$ & 396.0 & $\tan \beta$ & 10.0 \\
\hline$M_{H_{d}}$ & 159.8 & $\left|M_{H_{u}}\right|$ & 378.3 \\
\hline$M_{L_{1}}$ & 181.0 & $M_{L_{3}}$ & 179.3 \\
$M_{E_{1}}$ & 115.7 & $M_{E_{3}}$ & 110.0 \\
$M_{Q_{1}}$ & 525.8 & $M_{Q_{3}}$ & 471.4 \\
$M_{U_{1}}$ & 507.2 & $M_{U_{3}}$ & 387.5 \\
$M_{D_{1}}$ & 505.0 & $M_{D_{3}}$ & 500.9 \\
\hline
\end{tabular}

Table 3. The $\overline{\mathrm{DR}}$ SUSY Lagrangian parameters at the scale $\tilde{M}=1 \mathrm{TeV}$ in SPS1a' from [56] [mass unit in $\mathrm{GeV} ; M_{H_{u}}^{2}$ negative]. In addition, gauge and Yukawa couplings at this scale are given in the $\overline{\mathrm{DR}}$ scheme.

\begin{tabular}{|c||c|c|}
\hline Particle & Mass $[\mathrm{GeV}]$ & $\delta_{\text {scale }}[\mathrm{GeV}]$ \\
\hline \hline$h^{0}$ & 116.0 & 1.3 \\
$H^{0}$ & 425.0 & 0.7 \\
\hline$\tilde{\chi}_{1}^{0}$ & 97.7 & 0.4 \\
$\tilde{\chi}_{2}^{0}$ & 183.9 & 1.2 \\
$\tilde{\chi}_{4}^{0}$ & 413.9 & 1.2 \\
$\tilde{\chi}_{1}^{ \pm}$ & 183.7 & 1.3 \\
\hline$\tilde{e}_{R}$ & 125.3 & 1.2 \\
$\tilde{e}_{L}$ & 189.9 & 0.4 \\
$\tilde{\tau}_{1}$ & 107.9 & 0.5 \\
\hline$\tilde{q}_{R}$ & 547.2 & 9.4 \\
$\tilde{q}_{L}$ & 564.7 & 10.2 \\
$\tilde{t}_{1}$ & 366.5 & 5.4 \\
$\tilde{b}_{1}$ & 506.3 & 8.0 \\
\hline$\tilde{g}$ & 607.1 & 1.4 \\
\hline
\end{tabular}

Table 4. Supersymmetric masses for the SUSY scale $\tilde{M}=$ $1 \mathrm{TeV}$, and their variation if $\tilde{M}$ is shifted to $0.1 \mathrm{TeV}$.

yses of the $\beta$-functions as well as the other evolution coefficients (other codes can be used equally well).

This SPS1a' set is compatible with all high-energy mass bounds and with the low-energy precision data, as well as with the observed CDM data, calculated as $\mathcal{B}(b \rightarrow s \gamma)=3.0 \cdot 10^{-4}$ [57], $\Delta[g-2]_{\mu} / 2=34 \cdot 10^{-10}$ [58], $\Delta \rho_{\mathrm{SUSY}}=2.1 \cdot 10^{-4}\left[58\right.$, and $\Omega_{\mathrm{CDM}} h^{2}=0.10$ [57].

The physical [pole] masses of the supersymmetric particles are presented in Table 5 The connection between the Lagrangian parameters and the physical pole masses is presently encoded at the one-loop level for the masses of the SUSY particles, and at the two-loop level for the Higgs masses. QCD effects on the heavy quark masses are accounted for to two-loop accuracy.
A systematic comparison with the other public programs ISA JET [59, SOFTSUSY 60 and SuSpect 61 has been performed in 62 to estimate the technical accuracy that can presently be reached in the evolution. The codes include full two-loop RGEs for all parameters as well as one-loop formulas for threshold corrections. The agreement between the actual versions of these calculations is in general within one percent. A special case are the on-shell masses of the Higgs bosons which have been calculated by FeynHiggs [58 starting from the SPheno Lagrangian parameters as input. Here, discrepancies for the mass of the lightest Higgs boson amount to $2 \%$ or more which can be attributed to different renormalization schemes (see also 63 for detailed discussions).

Besides the comparison between different codes for spectrum calculations, a crude internal estimate of the theoretical errors at the present level of the loop calculations may be obtained by shifting the matching point $\tilde{M}$ from $1 \mathrm{TeV}$ down to $0.1 \mathrm{TeV}$. A sample of particle mass shifts associated with such a variation of the SUSY scale parameter is displayed in Table 4 With errors at the percent level, the experimental precision at LHC can be matched in general. However, it is obvious that another order of magnitude, the per-mil level, is required in the theoretical precision to match the expected experimental precision at ILC and in coherent LHC/ILC analyses - i.e., calculations of the next loop are called for ${ }^{1}$.

To perform experimental simulations, the branching ratios of the decay modes are crucial: these have been calculated using FeynHiggs [58] and SDECAY 65]; similar results may be obtained using CPSuperH [66]. The most important decay channels of the supersymmetric particles and Higgs bosons in SPS1a' are collected in the Appendix, while the complete set is available from the SPA web-site. Cross sections for the production of squarks, gluinos, gauginos and sleptons at the LHC are presented as a function of mass including the point SPS1a'. Typical cross sections for pair production of charginos, neutralinos and sleptons at the ILC are presented for the point $\mathrm{SPS1a}^{\prime}$ as a function of the collider energy.

If SPS1a', or a SUSY parameter set in the range of similar mass scales, is realized in nature, a plethora of interesting channels can be exploited to extract the basic supersymmetry parameters when combining experimental information from sharp edges in mass distributions at LHC with measurements of decay spectra and threshold excitation curves at an $e^{+} e^{-}$collider with energy up to $1 \mathrm{TeV}$ [11. From the simulated experimental errors the data analysis performed coherently for the two machines gives rise to a very precise picture of the supersymmetric particle spectrum as demonstrated in Table 6]

\footnotetext{
${ }^{1}$ With $\beta$ functions and evolution coefficients in the RGEs already available to third order 22, the calculation of the two-loop order for the relation between the Lagrangian parameters and the physical pole masses have been carried out in the approximation of massless vector bosons 64
} 


\begin{tabular}{|c|c||c|c|}
\hline Particle & Mass [GeV] & Particle & Mass $[\mathrm{GeV}]$ \\
\hline \hline$h^{0}$ & 116.0 & $\tilde{\tau}_{1}$ & 107.9 \\
$H^{0}$ & 425.0 & $\tilde{\tau}_{2}$ & 194.9 \\
$A^{0}$ & 424.9 & $\tilde{\nu}_{\tau}$ & 170.5 \\
$H^{+}$ & 432.7 & $\tilde{u}_{R}$ & 547.2 \\
\hline$\tilde{\chi}_{1}^{0}$ & 97.7 & $\tilde{u}_{L}$ & 564.7 \\
$\tilde{\chi}_{2}^{0}$ & 183.9 & $\tilde{d}_{R}$ & 546.9 \\
$\tilde{\chi}_{3}^{0}$ & 400.5 & $\tilde{d}_{L}$ & 570.1 \\
\cline { 3 - 4 }$\tilde{\chi}_{4}^{0}$ & 413.9 & $\tilde{t}_{1}$ & 366.5 \\
$\tilde{\chi}_{1}^{+}$ & 183.7 & $\tilde{t}_{2}$ & 585.5 \\
$\tilde{\chi}_{2}^{+}$ & 415.4 & $\tilde{b}_{1}$ & 506.3 \\
\cline { 1 - 2 }$\tilde{e}_{R}$ & 125.3 & $\tilde{b}_{2}$ & 545.7 \\
\cline { 3 - 4 }$\tilde{e}_{L}$ & 189.9 & $\tilde{g}$ & 607.1 \\
$\tilde{\nu}_{e}$ & 172.5 & & \\
\hline \multicolumn{3}{|c}{} \\
\hline
\end{tabular}

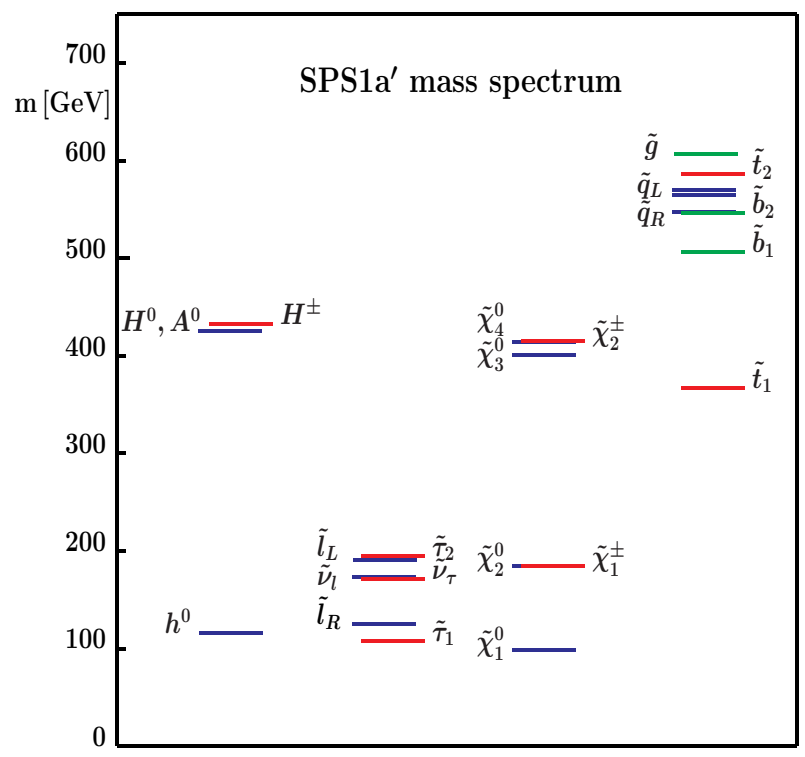

Table 5. Mass spectrum of supersymmetric particles [56] and Higgs bosons [58] in the reference point SPS1a'. The masses in the second generation coincide with the first generation.

\begin{tabular}{|c|c||c|c||c|}
\hline Particle & Mass & "LHC" & "ILC" & "LHC+ILC" \\
\hline \hline$h^{0}$ & 116.0 & 0.25 & 0.05 & 0.05 \\
$H^{0}$ & 425.0 & & 1.5 & 1.5 \\
\hline$\tilde{\chi}_{1}^{0}$ & 97.7 & 4.8 & 0.05 & 0.05 \\
$\tilde{\chi}_{2}^{0}$ & 183.9 & 4.7 & 1.2 & 0.08 \\
$\tilde{\chi}_{4}^{0}$ & 413.9 & 5.1 & $3-5$ & 2.5 \\
$\tilde{\chi}_{1}^{ \pm}$ & 183.7 & & 0.55 & 0.55 \\
\hline$\tilde{e}_{R}$ & 125.3 & 4.8 & 0.05 & 0.05 \\
$\tilde{e}_{L}$ & 189.9 & 5.0 & 0.18 & 0.18 \\
$\tilde{\tau}_{1}$ & 107.9 & $5-8$ & 0.24 & 0.24 \\
\hline$\tilde{q}_{R}$ & 547.2 & $7-12$ & - & $5-11$ \\
$\tilde{q}_{L}$ & 564.7 & 8.7 & - & 4.9 \\
$\tilde{t}_{1}$ & 366.5 & & 1.9 & 1.9 \\
$\tilde{b}_{1}$ & 506.3 & 7.5 & - & 5.7 \\
\hline$\tilde{g}$ & 607.1 & 8.0 & - & 6.5 \\
\hline
\end{tabular}

Table 6. Accuracies for representative mass measurements of SUSY particles in individual LHC, ILC and coherent "LHC+ILC" analyses for the reference point SPS1a' [mass units in $\mathrm{GeV}] . \tilde{q}_{R}$ and $\tilde{q}_{L}$ represent the flavors $q=u, d, c, s$. [Errors presently extrapolated from SPS1a simulations.]

While the picture so far had been based on evaluating the experimental observables channel by channel, global analysis programs have become available 67. 68 in which the whole set of data, masses, cross sections, branching ratios, etc. is exploited coherently to extract the Lagrangian parameters in the optimal way after including the available radiative corrections for masses and cross sections. With increasing numbers of observables the analyses can be expanded and refined in a systematic way. The present quality of such an analysis 68 can be judged from the results shown in Table 7 These errors are purely experimental and do not include the theoretical counterpart which must be improved considerably before matching the experimental standards.

\section{Extrapolation to the GUT scale}

Based on the parameters extracted at the scale $\tilde{M}$, we can approach the reconstruction of the fundamental supersymmetric theory and the related microscopic picture of the mechanism breaking supersymmetry. The experimental information is exploited to the maximum extent possible in the bottom-up approach 12 in which the extrapolation from $\tilde{M}$ to the GUT/Planck scale is performed by the renormalization group evolution for all parameters, with the GUT scale defined by the unification point of the two electroweak couplings. In this approach the calculation of loops and $\beta$ functions governing the extrapolation to the high scale is based on nothing but experimentally measured parameters. Typical examples for the evolution of the gaugino and scalar mass parameters are presented in Fig. 11 While the determination of the high-scale parameters in the gaugino/higgsino sector, as well as in the non-colored slepton sector, is very precise, the picture of the colored scalar and Higgs sectors is still coarse, and strong efforts should be made to refine it considerably.

On the other hand, if the structure of the theory at the high scale was known a priori and merely the experimental determination of the high-scale parameters were lacking, then the top-down approach would lead to a very precise parametric picture at the high scale. This is apparent from the fit of the mSUGRA parameters in SPS1a' displayed in Table 8 67. A high-quality fit of the parameters is a necessary condition, of course, 

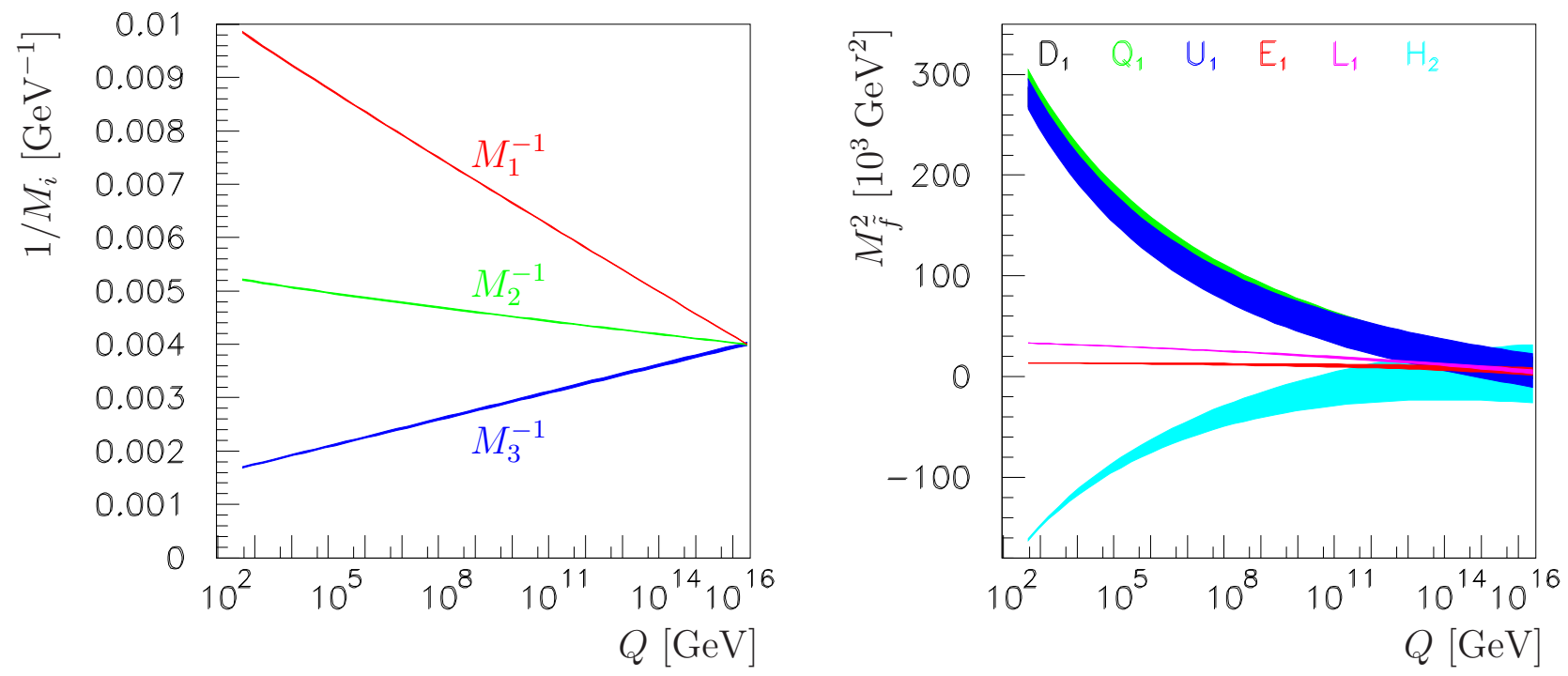

Fig. 1. Running of the gaugino and scalar mass parameters as a function of the scale $Q$ in SPS1a' [56]. Only experimental errors are taken into account; theoretical errors are assumed to be reduced to the same size in the future.

\begin{tabular}{|c||c|c|}
\hline Parameter & SPS1a $^{\prime}$ value & Fit error $[\mathrm{exp}]$ \\
\hline \hline$M_{1}$ & 103.3 & 0.1 \\
$M_{2}$ & 193.2 & 0.1 \\
$M_{3}$ & 571.7 & 7.8 \\
$\mu$ & 396.0 & 1.1 \\
\hline$M_{L_{1}}$ & 181.0 & 0.2 \\
$M_{E_{1}}$ & 115.7 & 0.4 \\
$M_{L_{3}}$ & 179.3 & 1.2 \\
\hline$M_{Q_{1}}$ & 525.8 & 5.2 \\
$M_{D_{1}}$ & 505.0 & 17.3 \\
$M_{Q_{3}}$ & 471.4 & 4.9 \\
\hline$m_{\mathrm{A}}$ & 372.0 & 0.8 \\
$A_{\mathrm{t}}$ & -565.1 & 24.6 \\
$\tan \beta$ & 10.0 & 0.3 \\
\hline
\end{tabular}

Table 7. Excerpt of extracted SUSY Lagrangian mass and Higgs parameters at the supersymmetry scale $\tilde{M}=1 \mathrm{TeV}$ in the reference point SPS1 $a^{\prime}$ [mass units in $\mathrm{GeV}$ ].

for the theory to be correct - however it is not a sufficient condition; deviations from the theory may hide in large errors of some observables which do not spoil the quality of the fit in the top-down approach but which are manifest in the bottom-up approach.

\section{$\underline{\text { Cold dark matter }}$}

Constraints on SUSY cold dark matter can be obtained at LHC by specifying the underlying scenario and analyzing all data simultaneously within the given benchmark model. From a study of the SPS1a point, based on very large statistics 69, one may expect that the relic density can be determined to $\sim 6 \%$ for the SPS1a ${ }^{\prime}$ scenario. For SPS1a', the relic density depends on the

\begin{tabular}{|c||c|c|}
\hline Parameter & SPS1a $^{\prime}$ value & Experimental error \\
\hline \hline$M_{\text {GUT }}$ & $2.47 \cdot 10^{16} \mathrm{GeV}$ & $0.02 \cdot 10^{16} \mathrm{GeV}$ \\
$\alpha_{\text {GUT }}^{-1}$ & 24.17 & 0.06 \\
\hline \hline$M_{\frac{1}{2}}$ & $250 \mathrm{GeV}$ & $0.2 \mathrm{GeV}$ \\
$M_{0}$ & $70 \mathrm{GeV}$ & $0.2 \mathrm{GeV}$ \\
$A_{0}$ & $-300 \mathrm{GeV}$ & $13.0 \mathrm{GeV}$ \\
\hline \hline$\mu$ & $396.0 \mathrm{GeV}$ & $0.3 \mathrm{GeV}$ \\
$\tan \beta$ & 10 & 0.3 \\
\hline \multicolumn{2}{|c}{} \\
\hline
\end{tabular}

Table 8. Comparison of the ideal parameters with the experimental expectations in the top-down approach [68].

parameters of the neutralino and sfermion sector as the dominant channels are annihilation of neutralinos into fermion pairs and coannihilation with staus. In particular, for the most sensitive component, coannihilation processes, the relic density is essentially given by the mass difference between the lightest slepton $\tilde{\tau}_{1}$ and the LSP $\tilde{\chi}_{1}^{0}$, which can be directly measured at the ILC. Studies of $\tilde{\tau}_{1}$ production at threshold 70 and decay spectra to $\tilde{\chi}_{1}^{0}$ in the continuum 71 suggest that for SPS1a', even with moderate luminosity, a precision of $\sim 2 \%$ on the cold dark matter abundance is achievable. A systematic analysis of various scenarios is being carried out in the LCC project [72] as well as by other groups.

\section{SUMMARY AND OUTLOOK}

If supersymmetry is realized in Nature, future experiments at the LHC and the ILC will provide very precise measurements of supersymmetric particle spectra and couplings. On the theoretical side these measurements 
must be matched by equally precise theoretical calculations and numerical analysis tools. The SPA Project, a joint theoretical and experimental effort, aims at providing

- a well-defined framework for SUSY calculations and data analyses,

- all necessary theoretical and computational tools,

- a testground scenario SPS1a',

- a platform for future extensions and developments.

On this basis coherent analyses of experimental data can be performed and the fundamental supersymmetric Lagrangian parameters can be extracted. They can serve as a firm base for extrapolations to high scales so that the ultimate supersymmetric theory and the supersymmetry breaking mechanism can be reconstructed from future data.

Much work is still needed on the experimental and theoretical side to achieve these goals at the desired level of accuracy. Some of the short- and long-term subprojects have been identified and should be pursued in the near future.

The SPA Project is a dynamical system expected to evolve continuously. The current status of the SPA Project, names of the conveners responsible for specific tasks as well as links to the available calculational tools, can be found at the SPA home page http://spa.desy.de/spa/

\section{APPENDIX}

\section{(a) Decays of Higgs and SUSY particles in SPS1a'}

The branching ratios of Higgs bosons and SUSY particles exceeding $2 \%$ are presented in Tables 912 The complete listing including all decays is available on the SPA web-site http://spa.desy.de/spa/

\begin{tabular}{|c||c||cc|cc|}
\hline Higgs & $m, \Gamma[\mathrm{GeV}]$ & decay & $\mathcal{B}$ & decay & $\mathcal{B}$ \\
\hline \hline$h^{0}$ & 116.0 & $\tau^{-} \tau^{+}$ & 0.077 & $W W^{*}$ & 0.067 \\
& $4 \times 10^{-3}$ & $b \bar{b}$ & 0.773 & $g g$ & 0.055 \\
& & $c \bar{c}$ & 0.021 & & \\
\hline$H^{0}$ & 425.0 & $\tau^{-} \tau^{+}$ & 0.076 & $\tilde{\chi}_{1}^{0} \tilde{\chi}_{2}^{0}$ & 0.038 \\
& 1.2 & $b \bar{b}$ & 0.694 & $\tilde{\chi}_{2}^{0} \tilde{\chi}_{2}^{0}$ & 0.020 \\
& & $t \bar{t}$ & 0.052 & $\tilde{\chi}_{1}^{+} \tilde{\chi}_{1}^{-}$ & 0.050 \\
& & $\tilde{\tau}_{1}^{ \pm} \tilde{\tau}_{2}^{\mp}$ & 0.030 & & \\
\hline$A^{0}$ & 424.9 & $\tau^{-} \tau^{+}$ & 0.057 & $\tilde{\chi}_{1}^{0} \tilde{\chi}_{2}^{0}$ & 0.054 \\
& 1.6 & $b \bar{b}$ & 0.521 & $\tilde{\chi}_{2}^{0} \tilde{\chi}_{2}^{0}$ & 0.060 \\
& & $t \bar{t}$ & 0.094 & $\tilde{\chi}_{1}^{+} \tilde{\chi}_{1}^{-}$ & 0.163 \\
& & $\tilde{\tau}_{1}^{ \pm} \tilde{\tau}_{2}^{\mp}$ & 0.036 & & \\
\hline$H^{+}$ & 432.7 & $\nu_{\tau} \tau^{+}$ & 0.104 & $\tilde{\chi}_{1}^{+} \tilde{\chi}_{1}^{0}$ & 0.143 \\
& 0.9 & $t \bar{b}$ & 0.672 & $\tilde{\nu}_{\tau} \tilde{\tau}_{1}^{+}$ & 0.071 \\
\hline
\end{tabular}

Table 9. Higgs masses and branching ratios $\mathcal{B}>2 \%$ in SPS1 $a^{\prime}$ from [58].

\begin{tabular}{|c||c||cc|cc|}
\hline$\tilde{\chi}$ & $m, \Gamma[\mathrm{GeV}]$ & decay & $\mathcal{B}$ & decay & $\mathcal{B}$ \\
\hline \hline$\tilde{\chi}_{1}^{0}$ & 97.7 & & & & \\
\hline$\tilde{\chi}_{2}^{0}$ & 183.9 & $\tilde{e}_{R}^{ \pm} e^{\mp}$ & 0.025 & $\tilde{\nu}_{e} \nu_{e}$ & 0.116 \\
& 0.083 & $\tilde{\tau}_{1}^{ \pm} \tau^{\mp}$ & 0.578 & $\tilde{\nu}_{\tau} \nu_{\tau}$ & 0.152 \\
\hline$\tilde{\chi}_{3}^{0}$ & 400.5 & $\tilde{\chi}_{1}^{ \pm} W^{\mp}$ & 0.582 & $\tilde{\chi}_{1}^{0} Z^{0}$ & 0.104 \\
& 2.4 & & & $\tilde{\chi}_{2}^{0} Z^{0}$ & 0.224 \\
\hline$\tilde{\chi}_{4}^{0}$ & 413.9 & $\tilde{\tau}_{2}^{ \pm} \tau^{\mp}$ & 0.033 & $\tilde{\chi}_{1}^{ \pm} W^{\mp}$ & 0.511 \\
& 2.9 & $\tilde{\nu}_{e} \nu_{e}$ & 0.042 & $\tilde{\chi}_{1}^{0} Z^{0}$ & 0.022 \\
& & $\tilde{\nu}_{\tau} \nu_{\tau}$ & 0.042 & $\tilde{\chi}_{2}^{0} Z^{0}$ & 0.024 \\
& & & & $\tilde{\chi}_{1}^{0} h^{0}$ & 0.070 \\
& & & & $\tilde{\chi}_{2}^{0} h^{0}$ & 0.165 \\
\hline \hline$\tilde{\chi}_{1}^{+}$ & 183.7 & $\tilde{\tau}_{1}^{+} \nu_{\tau}$ & 0.536 & $\tilde{\nu}_{\tau} \tau^{+}$ & 0.185 \\
& 0.077 & & & $\tilde{\nu}_{e} e^{+}$ & 0.133 \\
\hline$\tilde{\chi}_{2}^{+}$ & 415.4 & $\tilde{e}_{L}^{+} \nu_{e}$ & 0.041 & $\tilde{\chi}_{1}^{0} W^{+}$ & 0.063 \\
& 3.1 & $\tilde{\tau}_{2}^{+} \nu_{\tau}$ & 0.046 & $\tilde{\chi}_{2}^{0} W^{+}$ & 0.252 \\
& & $\tilde{t}_{1} b$ & 0.109 & $\tilde{\chi}_{1}^{+} Z^{0}$ & 0.221 \\
& & & & $\tilde{\chi}_{1}^{+} h^{0}$ & 0.181 \\
\hline
\end{tabular}

Table 10. Neutralino and chargino masses, widths and branching ratios $\mathcal{B}>2 \%$ in SPS1 $a^{\prime}$ from [65]; branching ratios for the second generation are the same as for the first generation. 


\begin{tabular}{|c||c||cc|cc|}
\hline$\tilde{\ell}$ & $m, \Gamma[\mathrm{GeV}]$ & decay & $\mathcal{B}$ & decay & $\mathcal{B}$ \\
\hline \hline$\tilde{e}_{R}$ & 125.3 & $\tilde{\chi}_{1}^{0} e^{-}$ & 1.000 & & \\
& 0.10 & & & & \\
\hline$\tilde{e}_{L}$ & 189.9 & $\tilde{\chi}_{1}^{0} e^{-}$ & 0.925 & $\tilde{\chi}_{1}^{-} \nu_{e}$ & 0.049 \\
& 0.12 & $\tilde{\chi}_{2}^{0} e^{-}$ & 0.026 & & \\
\hline$\tilde{\nu}_{e}$ & 172.5 & $\tilde{\chi}_{1}^{0} \nu_{e}$ & 1.000 & & \\
& 0.12 & & & & \\
\hline$\tilde{\tau}_{1}$ & 107.9 & $\tilde{\chi}_{1}^{0} \tau^{-}$ & 1.000 & & \\
& 0.016 & & & & \\
\hline$\tilde{\tau}_{2}$ & 194.9 & $\tilde{\chi}_{1}^{0} \tau^{-}$ & 0.868 & $\tilde{\chi}_{1}^{-} \nu_{\tau}$ & 0.086 \\
& 0.18 & $\tilde{\chi}_{2}^{0} \tau^{-}$ & 0.046 & & \\
\hline$\tilde{\nu}_{\tau}$ & 170.5 & $\tilde{\chi}_{1}^{0} \nu_{\tau}$ & 1.000 & & \\
& 0.12 & & & & \\
\hline
\end{tabular}

Table 11. Slepton masses, widths and branching ratios $\mathcal{B}>$ $2 \%$ in SPS1 $a^{\prime}$ from [65]; branching ratios for the second generation are the same as for the first generation.

\begin{tabular}{|c||c||cc|cc|}
\hline$\tilde{q}$ & $m, \Gamma[\mathrm{GeV}]$ & decay & $\mathcal{B}$ & decay & $\mathcal{B}$ \\
\hline \hline$\tilde{u}_{R}$ & 547.2 & $\tilde{\chi}_{1}^{0} u$ & 0.990 & & \\
& 1.2 & & & & \\
\hline$\tilde{u}_{L}$ & 564.7 & $\tilde{\chi}_{2}^{0} u$ & 0.322 & $\tilde{\chi}_{1}^{+} \bar{d}$ & 0.656 \\
& 5.5 & & & & \\
\hline$\tilde{d}_{R}$ & 546.9 & $\tilde{\chi}_{1}^{0} d$ & 0.990 & & \\
& 0.3 & & & & \\
\hline$\tilde{d}_{L}$ & 570.1 & $\tilde{\chi}_{2}^{0} d$ & 0.316 & $\tilde{\chi}_{1}^{-} \bar{u}$ & 0.625 \\
& 5.4 & & & & \\
\hline$\tilde{t}_{1}$ & 366.5 & $\tilde{\chi}_{1}^{0} t$ & 0.219 & $\tilde{\chi}_{1}^{+} b$ & 0.719 \\
& 1.5 & $\tilde{\chi}_{2}^{0} t$ & 0.062 & & \\
\hline$\tilde{t}_{2}$ & 585.5 & $\tilde{\chi}_{1}^{0} t$ & 0.042 & $\tilde{\chi}_{1}^{+} b$ & 0.265 \\
& 6.3 & $\tilde{\chi}_{2}^{0} t$ & 0.103 & $\tilde{\chi}_{2}^{+} b$ & 0.168 \\
& & & & $\tilde{t}_{1} Z^{0}$ & 0.354 \\
& & & & $\tilde{t}_{1} h^{0}$ & 0.059 \\
\hline$\tilde{b}_{1}$ & 506.3 & $\tilde{\chi}_{1}^{0} b$ & 0.037 & $\tilde{\chi}_{1}^{-} t$ & 0.381 \\
& 4.4 & $\tilde{\chi}_{2}^{0} b$ & 0.295 & $\tilde{t}_{1} W^{-}$ & 0.281 \\
\hline$\tilde{b}_{2}$ & 545.7 & $\tilde{\chi}_{1}^{0} b$ & 0.222 & $\tilde{\chi}_{1}^{-} t$ & 0.178 \\
& 1.0 & $\tilde{\chi}_{2}^{0} b$ & 0.131 & $\tilde{t}_{1} W^{-}$ & 0.401 \\
& & $\tilde{\chi}_{3}^{0} b$ & 0.028 & & \\
& & $\tilde{\chi}_{4}^{0} b$ & 0.038 & & \\
\hline$\tilde{g}$ & 607.1 & $\tilde{u}_{R} \bar{u}$ & 0.086 & $\tilde{t}_{1} \bar{t}$ & 0.189 \\
& 5.5 & $\tilde{u}_{L} \bar{u}$ & 0.044 & $\tilde{b}_{1} \bar{b}$ & 0.214 \\
& & $\tilde{d}_{R} \bar{d}$ & 0.087 & $\tilde{b}_{2} \bar{b}$ & 0.096 \\
& & $\tilde{d}_{L} \bar{d}$ & 0.034 & & \\
\hline
\end{tabular}

Table 12. Masses, widths and branching ratios $\mathcal{B}>2 \%$ of colored SUSY particles in SPS1 ' from [65]; branching ratios for the second generation are the same as for the first generation.

\section{(b) LHC and ILC cross sections in SPS1a'}

Total cross sections are presented in Figs. 2 - 6 for SUSY particle production at the LHC and the ILC.
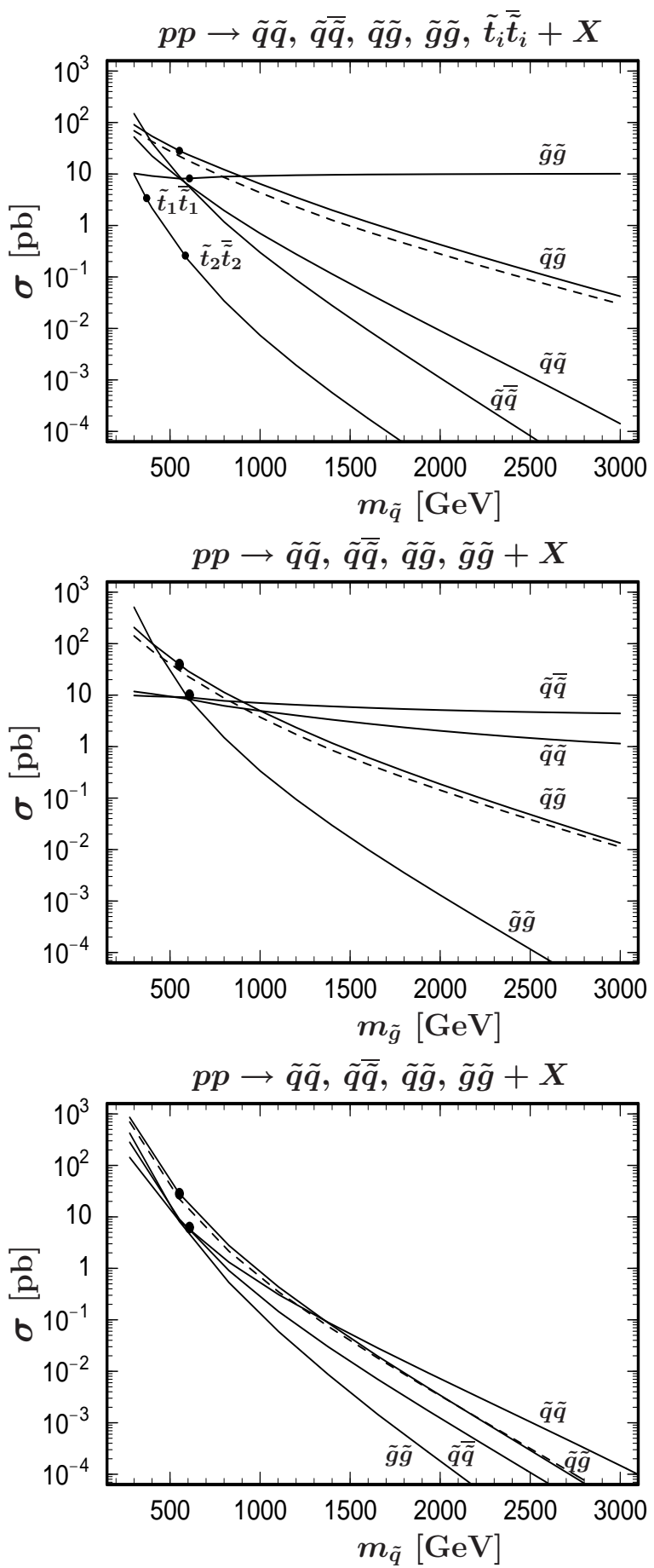

Fig. 2. Total cross sections for squark and gluino pair production at the LHC 27 28] for fixed gluino mass (top), squark mass (center), and gluino/squark mass ratio (bottom) [fixed parameters corresponding to SPS1a' values]. Black circles indicate the SPS1a' mass values. The Born cross sections (broken lines) are shown for some channels. 


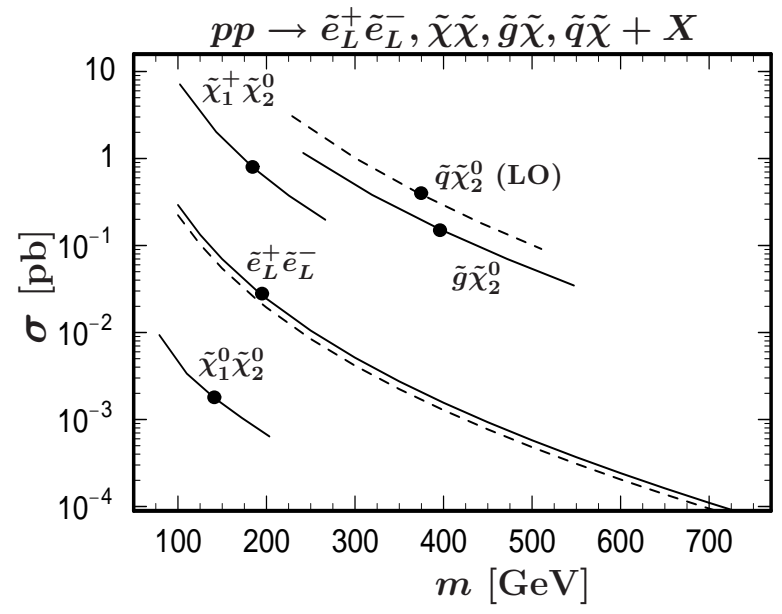

Fig. 3. Generic examples of total cross sections (DrellYan and Compton production) as a function of the average mass for production of sleptons, charginos and neutralinos at the LHC [27, 28]. The Born cross sections (broken line) are shown for comparison.
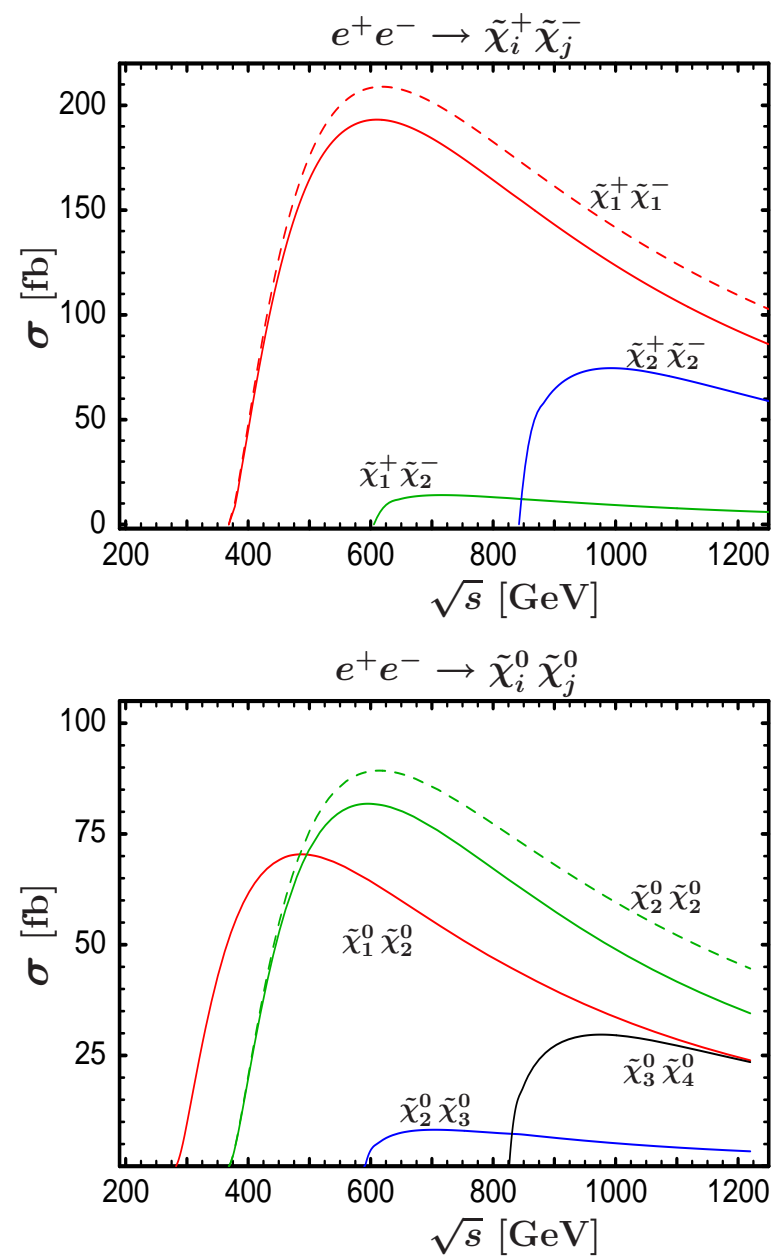

Fig. 4. Total cross section sections for chargino and neutralino pair production in $e^{+} e^{-}$annihilation [73]. The Born cross sections (broken lines) are shown for a few channels.
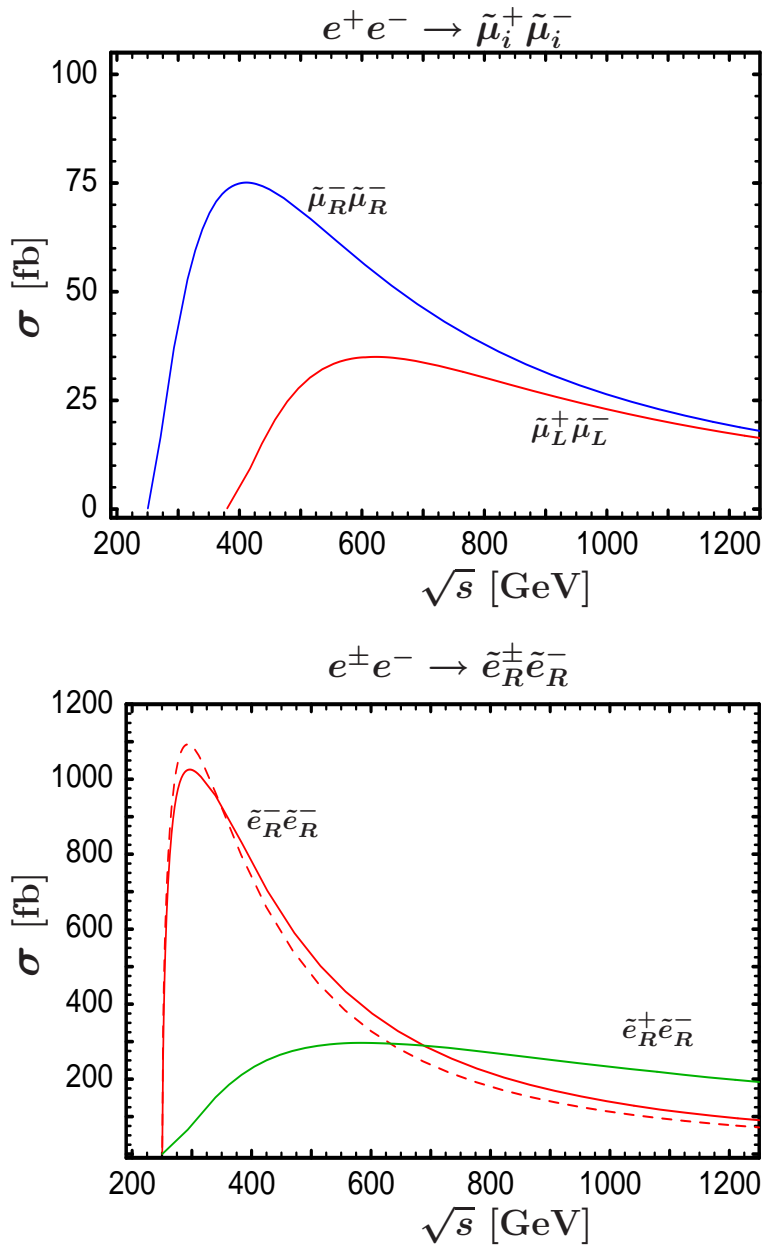

Fig. 5. Total cross sections for smuon and selectron pair production in $e^{ \pm} e^{-}$annihilation [74]. The Born cross section (broken lines) is shown for comparison.

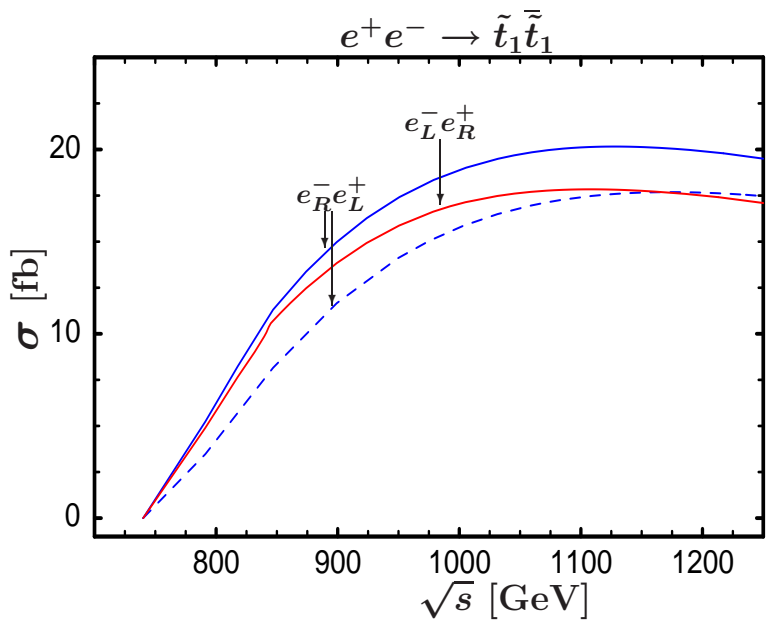

Fig. 6. Total cross sections for $\tilde{t}_{1} \overline{\tilde{t}}_{1}$ pair production in $e^{+} e^{-}$annihilation for left- and right-handed polarized electron $\left(P_{e^{-}}=0.8\right)$ and positron $\left(P_{e^{+}}=0.6\right)$ beams [75]. The Born cross section (broken line) is shown for comparison. 


\section{References}

1. J. Wess and B. Zumino, Nucl. Phys. B 70 (1974) 39.

2. H.-P. Nilles, Phys Rept. 110 (1984) 1; H. E. Haber and G. L. Kane, Phys. Rept. 117 (1985) 75.

3. J. Wess and J. Bagger, Supersymmetry and Supergravity, Princeton University Press, Princeton (1992).

4. M. Drees, R. M. Godbole and P. Roy, Theory and Phenomenology of Sparticles, World Scientifc, Singapore (2005).

5. ATLAS Technical Design Report, CERN/LHCC/9915, ATLAS TDR 15 (1999); CMS Technical Proposal, CERN/LHCC/94-38 (1994).

6. I. Hinchliffe et al., Phys. Rev. D 55, 5520 (1997); see contributions by B. K. Gjelsten et al., M. Chiorboli et al., J. Hisano et al. in $L H C+I L C$ Report 45].

7. H. Murayama and M. E. Peskin, Ann. Rev. Nucl. Part. Sci. 46 (1996) 533 [arXiv hep-ex/9606003; H. Baer, R. Munroe and X. Tata, Phys. Rev. D 54 (1996) 6735 [Erratum-ibid. D 56 (1997) 4424] [arXiv hep-ph/9606325; E. Accomando et al. [ECFA/DESY LC Physics Working Group], Phys. Rept. 299 (1998) 1 [arXiv hep-ph/9705442]; T. Behnke, J. D. Wells and P. M. Zerwas, Prog. Part. Nucl. Phys. 48 (2002) 363; S. Dawson and M. Oreglia, Ann. Rev. Nucl. Part. Sci. 54 (2004) 269 [arXiv hep-ph/0403015].

8. J.A. Aguilar-Saavedra et al. [ECFA/DESY LC Physics WG], TESLA Technical Design Report, DESY 01-011 and arXiv hep-ph/0106315 T. Abe et al. [American LC WG], SLAC-R-570 and arXiv hep-ex/0106055-58; K. Abe et al. [ACFA LC WG], KEK-Report-2001-011 and arXiv: hep-ph/0109166

9. H.-U. Martyn, ECFA/DESY LC Study, arXiv hep-ph/0406123 A. Freitas, H.-U. Martyn, U. Nauenberg and P. M. Zerwas, Proceedings International Linear Collider Conference LCWSO4, Paris 2004, arXiv hep-ph/0409129 J. K. Mizukoshi, H. Baer, A. S. Belyaev and X. Tata, Phys. Rev. D 64 (2001) 115017 [arXiv hep-ph/0107216.

10. CLIC Physics Working Group, arXiv hep-ph/0412251

11. B. C. Allanach et al., in $L H C+I L C$ Report [45], [and arXiv hep-ph/0403133 arXiv hep-ph/0407067.

12. G. A. Blair, W. Porod and P. M. Zerwas, Phys. Rev. D 63 (2001) 017703 [arXiv hep-ph/0007107 and Eur. Phys. J. C 27 (2003) 263 [arXiv hep-ph/0210058.

13. B. C. Allanach, D. Grellscheid and F. Quevedo, JHEP 0205 (2002) 048 [arXiv:0111057]; G. L. Kane, J. Lykken, S. Mrenna, B. D. Nelson. L. T. Wang and T. T. Wang, Phys. Rev. D 67 (2003) 045008 [arXiv hep-ph/0209061.

14. M. Chiorboli et al., in $L H C+I L C$ Report 45 .

15. D. J. H. Chung, L. L. Everett, G. L. Kane, S. F. King, J. Lykken and L. T. Wang, Phys. Rept. 407, 1 (2005) [arXiv hep-ph/0312378.

16. P. Skands et al., JHEP $0407 \quad$ (2004) 036 [arXiv hep-ph/0311123.

17. B. C. Allanach et al., Eur. Phys. J. C 25 (2002) 113 [arXiv hep-ph/0202233.

18. C. L. Bennett et al., Astrophys. J. Suppl. 148 (2003) 1 [arXiv astro-ph/0302207]. D. N. Spergel et al., Astrophys. J. Suppl. 148 (2003) 175 [arXiv astro-ph/0302209] and references therein.
19. M. Battaglia et al., Eur. Phys. J. C 33 (2004) 273 [arXiv hep-ph/0306219 and Eur. Phys. J. C 22 (2001) 535 [arXiv hep-ph/0106204.

20. W. Siegel, Phys. Lett. B 84 (1979) 193; D. M. Capper, D. R. T. Jones and P. van Nieuwenhuizen, Nucl. Phys. B 167 (1980) 479.

21. I. Jack, D. R. T. Jones, S. P. Martin, M. T. Vaughn and Y. Yamada, Phys. Rev. D 50 (1994) 5481 [arXiv hep-ph/9407291.

22. I. Jack, D. R. T. Jones and A. F. Kord, Annals Phys. 316 (2005) 213 [arXiv hep-ph/0408128; see also http://www.liv.ac.uk dij/betas/

23. D. Stöckinger, JHEP $0503 \quad$ (2005) [arXiv hep-ph/0503129.

24. W. Siegel, Phys. Lett. B 94 (1980) 37.

25. I. Jack and D. R. T. Jones, in Perspectives on Supersymmetry, World Scientific, ed. G. Kane, and arXiv hep-ph/9707278

26. W. Hollik, E. Kraus and D. Stöckinger, Eur. Phys. J. C 11, (1999) 365 [arXiv hep-ph/9907393; W. Hollik and D. Stöckinger, Eur. Phys. J. C 20 (2001) 105 [arXiv hep-ph/0103009; I. Fischer, W. Hollik, M. Roth and D. Stöckinger, Phys. Rev. D 69 (2004) 015004 [arXiv hep-ph/0310191.

27. W. Beenakker, R. Höpker, M. Spira and P. M. Zerwas, Phys. Rev. Lett. 74 (1995) 2905 [arXiv hep-ph/9412272, Z. Phys. C 69 (1995) 163 [arXiv hep-ph/9505416 and Nucl. Phys. B 492 (1997) 51 arXiv hep-ph/9610490]; W. Beenakker, M. Kramer, T. Plehn, M. Spira and P. M. Zerwas, Nucl. Phys. B 515 (1998) 3 [arXiv hep-ph/9710451; W. Beenakker, M. Klasen, M. Kramer, T. Plehn, M. Spira and P. M. Zerwas, Phys. Rev. Lett. 83 (1999) 3780 [arXiv hep-ph/9906298; E. L. Berger, M. Klasen and T. M. P. Tait, Phys. Rev. D 62 (2000) 095014; E: ibid. D 67 (2003) 099901 [arXiv hep-ph/0005196 and 0212306]; M. Spira, Proceedings 10th International Conference on Supersymmetry and Unification of Fundamental Interactions SUSY02, Hamburg (2002), arXiv hep-ph/0211145

28. W. Beenakker, R. Höpker and M. Spira, PROSPINO (version 2.0), arXiv hep-ph/9611232 see also 27.

29. A. Freitas and D. Stöckinger, Phys. Rev. D 66 (2002) 095014 [arXiv hep-ph/0205281.

30. J. F. Gunion, H. E. Haber, G. L. Kane and S. Dawson, Addison-Wesley, 1990; A. Djouadi, arXiv hep-ph/0503173

31. S. P. Martin and M. T. Vaughn, Phys. Lett. B 318 (1993) 331 [arXiv hep-ph/9308222.

32. A. Denner, Fortsch. Phys. 41 (1993) 307.

33. G. Degrassi, S. Fanchiotti and A. Sirlin, Nucl. Phys. B 351 (1991) 49; P. H. Chankowski, A. Dabelstein, W. Hollik, W. M. Mosle, S. Pokorski and J. Rosiek, Nucl. Phys. B 417 (1994) 101; K. Hagiwara, S. Matsumoto and Y. Yamada, Phys. Rev. Lett. 75 (1995) 3605.

34. S. Heinemeyer, W. Hollik and G. Weiglein, arXiv hep-ph/0412214 J. Haestier, S. Heinemeyer, D. Stöckinger and G. Weiglein, arXiv hep-ph/0508139

35. A. Freitas, W. Hollik, W. Walter and G. Weiglein, Nucl. Phys. B 632, 189 (2002) [Erratum-ibid. B 666, 305 (2003)] [arXiv hep-ph/0202131; M. Awramik and M. Czakon, Phys. Lett. B $\mathbf{5 6 8}$ (2003) 48 [arXiv hep-ph/0305248; M. Awramik, M. Czakon, A. Freitas and G. Weiglein, Phys. Rev. D 69053006 (2004) [arXiv hep-ph/0311148. 
36. D. M. Pierce, J. A. Bagger, K. T. Matchev and R. J. Zhang, Nucl. Phys. B 491 (1997) 3 [arXiv hep-ph/9606211.

37. H. Baer, J. Ferrandis, K. Melnikov and X. Tata, Phys. Rev. D 66 (2002) 074007 [arXiv hep-ph/0207126.

38. M. Carena, D. Garcia, U. Nierste and C. E. M. Wagner, Nucl. Phys. B 577 (2000) 88 [arXiv hep-ph/9912516.

39. W. Öller, H. Eberl and W. Majerotto, Phys. Rev. D 71 (2005) 115002 [arXiv hep-ph/0504109; A. Freitas, DESY-THESIS-2002-023 [DESY Document Server], see also Eur. Phys. J. C34 (2004) 487 [arXiv hep-ph/0310182; T. Fritzsche and W. Hollik, Nucl. Phys. Proc. Suppl. 135 (2004) 102 [arXiv hep-ph/0407095].

40. H. L. Lai et al., Eur. Phys. J. C 12 (2000) 375 [arXiv hep-ph/9903282.

41. A. D. Martin, R. G. Roberts, W. J. Stirling and R. S. Thorne, Eur. Phys. J. C $23 \quad 73 \quad$ (2002) [arXiv hep-ph/0110215.

42. Z. Kunszt, A. Signer and Z. Trocsanyi, Nucl. Phys. B 411 (1994) 397 [arXiv hep-ph/9305239]; S. Catani, M. H. Seymour and Z. Trocsanyi, Phys. Rev. D 55 (1997) 6819 [arXiv hep-ph/9610553.

43. A. Signer and D. Stöckinger, Phys. Lett. B 626 (2005) 127 [arXiv hep-ph/0508203.

44. W. Porod, in preparation.

45. LHC/LC Study Group, G. Weiglein et al., $L H C+I L C$ Report, arXiv hep-ph/0410364 submitted to Phys. Rept.

46. A. Djouadi, M. Drees and J. L. Kneur, JHEP 0108 (2001) 055 [arXiv hep-ph/0107316.

47. G. Bélanger, F. Boudjema, A. Cottrant and A. Pukhov, LAPTH-1052-04 [arXiv hep-ph/0407218; H. Baer and C. Balazs, JCAP 0305 (2003) 006 [arXiv hep-ph/0303113; H. Baer, C. Balazs, A. Belayev, R. Dermisen, A. Mafi and A. Mustafayev, JHEP 0205 (2002) 061 [arXiv hep-ph/0204108; J. R. Ellis, K. A. Olive, Y. Santoso and V. C. Spanos, Phys. Lett. B 565 (2003) 176 [arXiv hep-ph/0303043].

48. B. C. Allanach, G. Bélanger, F. Boudjema and A. Pukhov, JHEP $0412020 \quad$ (2004) [arXiv hep-ph/0410091; J. R. Ellis, K. A. Olive, Y. Santoso and V. C. Spanos, Phys. Lett. B 603 (2004) 51 [arXiv hep-ph/0408118.

49. H.E. Haber, in "Review of particle physics" [Particle Data Group], Phys. Lett. B 592 (2004) 1.

50. S. F. King, Rept. Prog. Phys. 67 (2004) 107 [arXiv hep-ph/0310204; H. Baer, C. Balazs, J. K. Mizukoshi and X. Tata, Phys. Rev. D 63 (2001) 055011 [arXiv hep-ph/0010068; A. Freitas, W. Porod and P. M. Zerwas, arXiv hep-ph/0509056

51. K. Desch, J. Kalinowski, G. Moortgat-Pick, M. M. Nojiri and G. Polesello, JHEP 0402 (2004) 035 [arXiv hep-ph/0312069 and arXiv hep-ph/0410121

52. J. A. Aguilar-Saavedra, arXiv hep-ph/0312140

53. M. Berggren, Proceedings International Linear Collider Conference LCWS04, Paris (2004), arXiv hep-ph/0508247

54. K. Kawagoe, M. M. Nojiri and G. Polesello, Phys. Rev. D 71 (2005) 035008 [arXiv hep-ph/0410160].

55. J. Ellis, K. A. Olive, Y. Santoso and V. C. Spanos, Phys. Rev. D 70 (2004) 055005 [arXiv hep-ph/0405110].
56. W. Porod, SPheno (version 2.2.3), Comput. Phys. Commun. 153 (2003) 275 [arXiv hep-ph/0301101.

57. G. Bélanger, F. Boudjema, A. Pukhov and A. Semenov, MicrOMEGAs (version 1.4), arXiv hep-ph/0405253 see also P. Gondolo, J. Edsjo, P. Ullio, L. Bergstrom, M. Schelke and E. A. Baltz, JCAP 0407, 008 (2004) [arXiv astro-ph/0406204]; H. Baer, C. Balazs, A. Belyaev and J. O'Farrill, JCAP 0309 (2003) 007 [arXiv hep-ph/0305191 and H. Baer, Proceedings International Linear Collider Conference LCWSO4, Paris (2004).

58. S. Heinemeyer, W. Hollik and G. Weiglein, FeynHiggs (version 2.2.10), Comput. Phys. Commun. 124 (2000) 76 [arXiv hep-ph/9812320]; see also S. Heinemeyer, W. Hollik and G. Weiglein, Eur. Phys. J. C 9 (1999) 343 [arXiv hep-ph/9812472] G. Degrassi, S. Heinemeyer, W. Hollik, P. Slavich and G. Weiglein, Eur. Phys. J. C 28 (2003) 133 [arXiv hep-ph/0212020].

59. F. E. Paige, S. D. Protopescu, H. Baer and X. 'Tata, ISAJET (version 7.71), arXiv hep-ph/0312045 H. Baer, J. Ferrandis, S. Kraml and W. Porod, arXiv hep-ph/0511123

60. B. C. Allanach, SOFTSUSY (version 2.0), Comput. Phys. Commun. $143 \quad$ (2002) 305 [arXiv hep-ph/0104145.

61. A. Djouadi, J. L. Kneur and G. Moultaka, SuSpect (version 2.3.4), arXiv hep-ph/0211331

62. B. C. Allanach, S. Kraml and W. Porod, JHEP 0303 (2003) 016 [arXiv hep-ph/0302102; G. Belanger, S. Kraml and A. Pukhov, Phys. Rev. D 72 (2005) 015003 [arXiv hep-ph/0502079; see also http://kraml.home.cern.ch/kraml/comparison/

63. M. Carena, H. E. Haber, S. Heinemeyer, W. Hollik, C. E. M. Wagner and G. Weiglein, Nucl. Phys. B 580 (2000) 29 [arXiv hep-ph/0001002 ; B. C. Allanach, A. Djouadi, J. L. Kneur, W. Porod and P. Slavich, JHEP 0409 (2004) 044 [arXiv hep-ph/0406166.

64. S. P. Martin, Phys. Rev. D 71 (2005) 116004 [arXiv hep-ph/0502168 and arXiv hep-ph/0509115 Y. Yamada, Phys. Lett. B $\mathbf{6 2 3} \quad(2005)$ [arXiv hep-ph/0506262.

65. M. Mühlleitner, A. Djouadi and Y. Mambrini, SDECAY (version 1.1a), arXiv hep-ph/0311167

66. J. S. Lee, A. Pilaftsis, M. Carena, S. Y. Choi, M. Drees, J. R. Ellis and C. E. M. Wagner, Comput. Phys. Commun. 156 (2004) 283 [arXiv hep-ph/0307377.

67. R. Lafaye, T. Plehn and D. Zerwas, SFITTER, arXiv hep-ph/0404282

68. P. Bechtle, K. Desch and P. Wienemann, FITTINo, arXiv hep-ph/0412012 accepted by Comput. Phys. Commun.; for recent developments see also P. Bechtle, Proceedings 2005 International Linear Collider Workshop LCWS05, Stanford (2005), arXiv hep-ph/0506244 P. Bechtle, K. Desch, W. Porod and P. Wienemann, arXiv hep-ph/0511006

69. G. Polesello and D. R. Tovey, JHEP 0405 (2004) 071 [arXiv hep-ph/0403047.

70. P. Bambade, M. Berggren, F. Richard and Z. Zhang, Proceedings International Linear Collider Conference LCWS04, Paris (2004), arXiv hep-ph/0406010

71. H.-U. Martyn, ECFA/DESY LC Study and contribution to International Linear Collider Conference LCWS04, Paris (2004), arXiv hep-ph/0408226 V. Khotilovich, R. Arnowitt, B. Dutta and T. Kamon, Phys. Lett. B 618 (2005) 182 [arXiv hep-ph/0503165]. 
72. LCC study group, http://physics.syr/edu/ trodden/lc-cosmology/

73. T. Fritzsche and W. Hollik, Nucl. Phys. Proc. Suppl. 135 (2004) 102 [arXiv hep-ph/0407095; W. Öller, H. Eberl and W. Majerotto, Phys. Rev. D 71 (2005) 115002 [arXiv hep-ph/0504109 and Phys. Lett. B 590 (2004) 273 [arXiv hep-ph/0402134.

74. A. Freitas, A. von Manteuffel and P. M. Zerwas, Eur. Phys. J. C 34 (2004) 487 [arXiv hep-ph/0310182

75. K. Kovarik, C. Weber, H. Eberl and W. Majerotto, Phys. Rev. D $\mathbf{7 2} \quad$ (2005) 053010 [arXiv hep-ph/0506021 and Phys. Lett. B 591 (2004) 242 [arXiv hep-ph/0401092; A. Arhrib and W. Hollik, JHEP 0404 (2004) 073 [arXiv hep-ph/0311149. 